Research Article

\title{
Exact Solutions for Interaction of Parallel Screw Dislocations with a Wedge Crack in One-Dimensional Hexagonal Quasicrystal with Piezoelectric Effects
}

\author{
Xin Lv $\mathbb{D}^{1,2}$ and Guan-Ting Liu $\mathbb{1}^{1}$ \\ ${ }^{1}$ College of Mathematics Science, Inner Mongolia Normal University, Hohhot 010022, China \\ ${ }^{2}$ School of Mechanical Engineering, Tianjin University, Tianjin 300350, China \\ Correspondence should be addressed to Guan-Ting Liu; guantingliu@imnu.edu.cn
}

Received 7 October 2019; Revised 13 April 2020; Accepted 21 April 2020; Published 29 May 2020

Academic Editor: Francesco Aymerich

Copyright (c) 2020 Xin Lv and Guan-Ting Liu. This is an open access article distributed under the Creative Commons Attribution License, which permits unrestricted use, distribution, and reproduction in any medium, provided the original work is properly cited.

\begin{abstract}
The purpose of this paper is to consider the interaction between many parallel dislocations and a wedge-shaped crack and their collective response to the external applied generalized stress in one-dimensional hexagonal piezoelectric quasicrystal, employing the complex variable function theory and the conformal transformation method; the problem for the crack is reduced to the solution of singular integral equations, which can be further reduced to solving Riemann-Hilbert boundary value problems. The analytical solutions of the generalized stress field are obtained. The dislocations are subjected to the phonon field line force, phason field line force, and line charge at the core. The positions of the dislocations are arbitrary, but the dislocation distribution is additive. The dislocation is not only subjected to the external stress and the internal stress generated by the crack, but also to the force exerted on it by other dislocations. The closed-form solutions are obtained for field intensity factors and the image force on a screw dislocation in the presence of a wedge-shaped crack and a collection of other dislocations. Numerical examples are provided to show the effects of wedge angle, dislocation position, dislocation distribution containing symmetric configurations and dislocation quantities on the field intensity factors, energy release rate, and image force acting on the dislocation. The principal new physical results obtained here are (1) the phonon stress, phason stress, and electric displacement singularity occur at the crack tip and dislocations cores, (2) the increasing number of dislocations always accelerates the crack propagation, (3) the effect of wedge angle on crack propagation is related to the distribution of dislocations, and (4) the results of the image force on the dislocation indicate that the dislocations can either be attracted or rejected and reach stable positions eventually.
\end{abstract}

\section{Introduction}

As a new structure of solid, quasicrystal (QC) was discovered by Shechtman et al. [1] in 1984. QC is a new solid structure with long-range orientation order and without translational symmetry which differs from crystals. The elastic problem of QC is related not only to the phonon field which describes lattice vibration, but also to the phason field which is about the quasiperiodic arrangement of atoms. As soon as the quasicrystal was discovered, it attracted wide attention because of its special symmetry and good performance. In the early discovery of the quasicrystal, the defects in the quasicrystal were observed [2]. Due to the unique properties of quasicrystal materials, many achievements have been made in the study of quasicrystal elasticity and fracture mechanics [3-9]. To name a few recent researches, Lou et al. [10] proposed a model of a thin elastic inclusion embedded in an infinite 1D hexagonal quasicrystal. Wang and Schiavone [11] investigated the elastic field near the tip of an anticrack in a homogeneous decagonal quasicrystal material subjected to plane strain deformations. Zhao et al. [12] studied the frictional contact problem and the adhesive contact problem in three-dimensional (3D) icosahedral quasicrystal. Li et al. [13] have given analytical solutions for arbitrarily shaped planar cracks in a two-dimensional (2D) hexagonal QC media. 
Quasicrystal is sensitive to force, electricity, magnetism, heat, and light, and these physical properties have been extensively studied. It is well known that QCs with piezoelectric effects have better performance in smart composite structures. Therefore, the study of quasicrystal materials considering the piezoelectric effect is of great significance. A lot of work on QC with piezoelectric effects has been published in the open literature. Li et al. [14] obtained threedimensional general solutions to static problems of a 1D hexagonal piezoelectric QC. Yu et al. [15] considered an antiplane crack problem by the semi-inverse method in a $1 \mathrm{D}$ hexagonal piezoelectric QC. Yu et al. [16] also discussed the complex variable method for an antiplane elliptical cavity. Yang et al. [17] presented the fracture behavior of two asymmetrical limited permeable cracks emanating from an elliptical hole in a one-dimensional hexagonal quasicrystal with piezoelectric effects. Yang et al. [18] derived the closedform solution for a multilayered two-dimensional decagonal QC plate. Guo et al. [19] analyzed an elliptical inclusion embedded in an infinite $1 \mathrm{D}$ hexagonal piezoelectric QC matrix. Li et al. [20] have given the exact solution for a layered piezoelectric quasicrystal nanoplate with nonlocal effect in cylindrical bending. Zhang et al. [21] addressed the static bending deformation of a functionally graded multilayered 1D hexagonal piezoelectric QC simply supported nanoplate. Zhou and $\mathrm{Li}$ [22] dealt with the problem of two collinear cracks in a one-dimensional hexagonal piezoelectric QC strip under mode III far-field loading, while they analyzed the electroelastic problem related to two collinear cracks of equal length and normal to the boundaries of a $1 \mathrm{D}$ hexagonal piezoelectric quasicrystal layer with the aid of a singular integral equation in ref. [23]. Besides, Zhou and Li [24] examined the electroelastic behavior induced by a penny-shaped dielectric crack of a 1D hexagonal piezoelectric quasicrystal plate of finite thickness, and they considered a Yoffe-type moving crack in a 1D hexagonal piezoelectric quasicrystal by using the Fourier transform technique in ref. [25]. Li and Liu [26] derived the electroelastic field of a straight dislocation in an icosahedral piezoelectric QC. Li et al. [27] displayed the interaction between a screw dislocation and an elliptical hole with two asymmetrical cracks in a $1 \mathrm{D}$ hexagonal quasicrystal with piezoelectric effects. $\mathrm{Hu}$ et al. [28] studied an antiplane interface crack between dissimilar 1D hexagonal piezoelectric QC.

Engineering materials always contain some defects, such as dislocations, inclusions, and cracks. The existence of these defects often seriously affects the safety and reliability of materials. There is not only one defect in the material, but usually different types of defects also exist at the same time, and the interaction between multiple defects has a very important impact on the mechanical properties and physical properties of the material. Dislocation activity is the cause of plastic deformation at the crack tip, which determines the crack propagation behavior, so the interaction between crack and dislocation has attracted more and more attention. Concerning the wedge-shaped crack which is an important geometric form of structures interacting with a single dislocation, Ohr et al. [29] performed the elastic analysis of the interaction between a wedge crack and a screw dislocation. Zhang et al. [30] obtained an analytical solution of the interaction of edge dislocation with the wedge crack by using the elastic technique of Muskhelishvili. Chen et al. [31] derived the closed solution of the electroelastic field for a screw dislocation situated near the tip two-bonded wedge-shaped dissimilar piezoelectric materials. Furthermore, they deduced the electroelastic stress field in the interaction of a piezoelectric screw dislocation with a wedge-shaped bimaterial interface in ref. [32]. Li and Zhao [33] coped with the problem of the interaction of a screw dislocation with the interface and wedge-shaped cracks in a one-dimensional hexagonal piezoelectric QC biomaterial using the conformal mapping method in conjunction with the image principle. Jiang and Liu [34] investigated the interaction between a screw dislocation and a wedge-shaped crack in the $1 \mathrm{D}$ hexagonal piezoelectric QC based on the conformal mapping method and the perturbation technique.

The research studies mentioned above were mainly focused on the single screw dislocation interacting with the various types of cracks. In engineering practice, structures with pairs of dislocations are very common. Dislocation emission and dislocation pile-up at the crack tip will result in crack blunting, inhibiting the growth of the crack and then improving the fracture toughness of the material. Concerning multiple dislocations interacting with cracks, the dislocation is not only subjected to the external stress and the internal stress generated by the crack, but also to the force exerted on it by other dislocations. Lin and Thomson [35] earlier investigated the elastic interaction between pairs of dislocations and a cleavage crack under external loads. Later, Juang and Lee [36] examined the interaction of the two-dislocation model and a surface crack by using the dislocationmodelling technique and the complex variable method. More recently, Liu and Yang [37] made a detailed analysis of the stress field of infinitely many parallel screw dislocations near a semi-infinite crack tip without applied loads, and dislocations considered here are located at particular points to make the total stress finite. Lv and Liu [38] addressed the analytical solution of the generalized stress field for parallel screw dislocations near a semiinfinite crack in magnetic-electric-mechanical coupling fields. However, for the piezoelectric QC, these problems become more complicated than the conventional material due to the introduction of the phason field and the electric field. To the best of the authors' knowledge, the study on the interaction between the wedge-shaped crack and multiple dislocations in a $1 \mathrm{D}$ hexagonal QC with piezoelectric effect has not been reported yet. In this paper, through the singular integral technique, we consider the antiplane problem of the interaction between many parallel screw dislocations and a wedge-shaped crack under remote uniform loadings, and the dislocations are subjected to the phonon field line force, phason field line force, and line charge at the core. The positions of the dislocations are arbitrary. The advantage of the present 
model is demonstrated when dealing with many dislocations because the dislocation distribution is additive. The expressions for the generalized stress field, field intensity factors, energy release rate, and image force are derived in a closed form. The exact solutions are presented and compared with those already obtained by other authors. Numerical examples are presented for various parameters of the problem.

\section{Basic Equations}

The quasicrystal piezoelectric material considered here is transversely isotropic with a hexagonal symmetry, which has an isotropic basal plane of $x_{1}-x_{2}$ plane and an electric field poling direction and quasiperiodic direction of the $x_{3}$ axis. When the defects penetrate along the $x_{3}$ axis, the quasicrystal piezoelectric boundary value problem is treated as in the case of out-of-plane phonon field displacement $u_{3}\left(x_{1}, x_{2}\right)$, out-of- plane phason field displacement $w_{3}\left(x_{1}, x_{2}\right)$, and in-plane electric field $\varphi\left(x_{1}, x_{2}\right)$. The basic equations can be written as follows [39].

In this case, the constitutive relations become

$$
\begin{aligned}
\sigma_{3 k} & =C_{44} u_{3, k}+R_{3} w_{3, k}-e_{15} \varphi_{, k}, \\
H_{3 k} & =R_{3} u_{3, k}+K_{2} w_{3, k}-d_{15} \varphi_{, k}, \\
D_{k} & =e_{15} u_{3, k}+d_{15} w_{3, k}+\lambda_{11} \varphi_{, k}, \\
(k & =1,2) .
\end{aligned}
$$

Generalized strain-displacement relations are given by

$$
\begin{aligned}
& \varepsilon_{i j}=\frac{\left(u_{i, j}+u_{j, i}\right)}{2}, \\
& \omega_{i j}=w_{i, j}, \\
& E_{i}=-\phi_{, i}, \\
& (i=1,2,3) .
\end{aligned}
$$

Equilibrium equations are

$$
\begin{aligned}
\sigma_{i j, i} & =0, \\
H_{i j, i} & =0, \\
D_{i, i} & =0,
\end{aligned}
$$

where a comma represents differentiation with respect to $x_{i}(i=1,2,3)$; the repeated indices denote that summation $\sigma_{3 k}$ and $\varepsilon_{3 k}$ are the phonon field stress and strain; $H_{3 k}$ and $\omega_{3 k}$ are the phason field stress and strain; $D_{k}$ and $E_{k}$ are the electric displacement and the electric field; $C_{44}, K_{2}$, and $R_{3}$ are the phonon field elastic, phason field elastic, and phonon-phason field coupling coefficients; $e_{15}$ and $d_{15}$ are the piezoelectric constants; $\lambda_{11}$ is the dielectric constant.

Substituting equations (1) and (2) to (3), the governing equation becomes

$$
C \nabla^{2} \mathbf{u}=0
$$

in which

$$
\begin{aligned}
u & =\left[\begin{array}{lll}
u_{3} & w_{3} & \varphi
\end{array}\right]^{T}, \\
\nabla^{2} & =\frac{\partial^{2}}{\partial x_{1}^{2}}+\frac{\partial^{2}}{\partial x_{2}^{2}}, \\
\mathbf{C} & =\left[\begin{array}{lll}
C_{44} & R_{3} & e_{15} \\
R_{3} & K_{2} & d_{15} \\
e_{15} & d_{15} & -\lambda_{11}
\end{array}\right] .
\end{aligned}
$$

In general,

$\operatorname{det} \mathbf{C}=-C_{44} d_{15}^{2}-K_{2} e_{15}^{2}+2 R_{3} d_{15} e_{15}-C_{44} K_{2} \lambda_{11}+R_{3}^{2} \lambda_{11} \neq 0$.

Equation (4) can be written as

$$
\nabla^{2} \mathbf{u}=0
$$

\section{Description of the Problem and Its Solutions}

3.1. The Electroelastic field of Parallel Screw Dislocations. The physical problem is shown in Figure 1. There are $n$ parallel screw dislocations located at $z_{i}\left(r_{i}, \theta_{i}\right)(i=1,2, \ldots, n)$ with the Burgers vectors $\left(0,0, b_{3}^{(i)}, b_{\perp}^{(i)}, b_{\varphi}^{(i)}\right)$ in the vicinity of a wedge-shaped crack in the $1 \mathrm{D}$ hexagonal QC piezoelectric material which is under the far-field uniform loading $\left(\sigma_{32}^{\infty}, H_{32}^{\infty}, E_{2}^{\infty}\right)$. The dislocations are subjected to the phonon field line force $p$, phason field line force $q$, and line charge $v$ at the core. The wedge-shaped crack is infinitely long along the negative real axis, and $\alpha$ denotes the wedge angle.

As shown in Figure 1, we have the following crack boundary conditions:

$$
\begin{aligned}
\sqrt{x_{1}^{2}+x_{2}^{2}} \longrightarrow \infty: \sigma_{32} & =\sigma_{32}^{\infty}, \\
H_{32} & =H_{32}^{\infty}, E_{2}=E_{2}^{\infty}, \\
(x, y) \in \theta & = \pm\left(\pi-\frac{\alpha}{2}\right): \sigma_{32}=0, \\
H_{32} & =0, \\
E_{2} & =0 .
\end{aligned}
$$

Proposing a conformal transformation function

$$
\begin{aligned}
& z=m(\zeta)=\zeta^{1 / \lambda}, \\
& \lambda=\frac{2 \pi}{2 \pi-\alpha},
\end{aligned}
$$

which maps the wedge-shaped crack in the $z$ plane to a semi-infinite crack along the negative real axis in the $\zeta$ plane (see Figure 2). The problem turns into the interaction of parallel screw dislocations with a semi-infinite crack. 


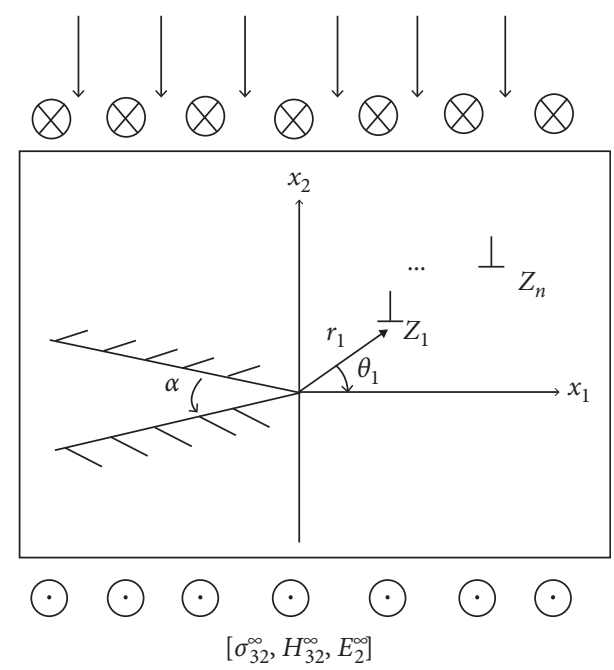

FIGURE 1: The interaction between a wedge-shaped crack and many parallel screw dislocations.

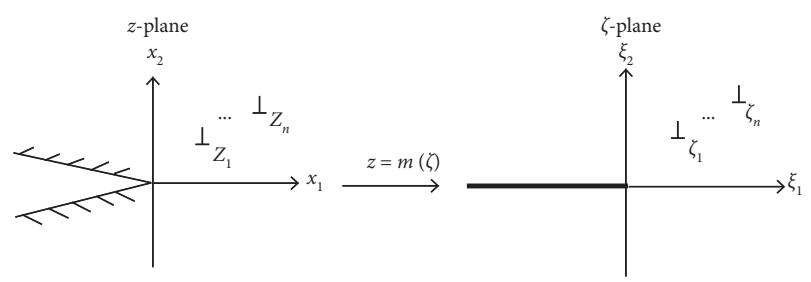

Figure 2: Conformal transformation.

In the $\zeta$ plane, we first consider the analytical solution of the elastic field of a screw dislocation. Assuming that one of the dislocations is situated at the point $\zeta_{d}$ with the Burgers vector $\left(0,0,0, b_{3}, b_{\perp}, b_{\varphi}\right)$, $\mathbf{u}$ can be expressed by the imaginary part of an arbitrary analytic function $\mathbf{f}(\zeta)$ following the theory of a complex variable function, and $\zeta=\xi_{1}+\xi_{2}$ and

$$
\mathbf{u}=\operatorname{Im}[\mathbf{f}(\zeta)]
$$

It is reasonable to assume that the potential function is composed of two parts; that is,

$$
\mathbf{f}(\zeta)=\mathbf{f}_{0}(\zeta)+\mathbf{f}_{1}(\zeta)
$$

where $\mathbf{f}_{0}(\zeta)$ is the complex potential for the dislocation in an infinite medium and $\mathbf{f}_{1}(\zeta)$ is the additional term which assumes that the stress boundary conditions on the cleavage surfaces are satisfied. Based on the force conditions of the dislocation, $\mathbf{f}_{0}(\zeta)$ can be chosen as

$$
\mathbf{f}_{0}(\zeta)=\mathbf{A} \ln \left(\zeta-\zeta_{d}\right),
$$

with

$$
\mathbf{A}=\left[A_{1}+\mathrm{i} A_{2}, B_{1}+\mathrm{i} B_{2}, C_{1}+\mathrm{i} C_{2}\right]^{\mathrm{T}} .
$$

Then,

$$
\begin{aligned}
\operatorname{Im}\left[\oint_{\zeta_{d}} \mathbf{f}_{0}^{\prime}(\zeta) \mathrm{d} \zeta\right] & =\operatorname{Im}\left[\oint_{\zeta_{d}} \frac{\mathbf{A}}{\zeta-\zeta_{d}} \mathrm{~d} \zeta\right]=\operatorname{Im}[2 \pi \mathrm{iA}] \\
& =\left[b_{3}, b_{\perp}, b_{\varphi}\right]^{\mathrm{T}} \\
\operatorname{Im}\left[\oint_{\zeta_{d}}-i \mathbf{C} \mathbf{f}_{0}^{\prime}(\zeta) \mathrm{d} \zeta\right] & =\operatorname{Im}\left[\oint_{\zeta_{d}} \frac{-i \mathbf{C A}}{\zeta-\zeta_{d}} \mathrm{~d} \zeta\right]=\operatorname{Im}[2 \pi \mathbf{C A}] \\
& =[-p,-q, v]^{\mathrm{T}} .
\end{aligned}
$$

From equations (10)-(14), we have

$$
\left\{\begin{array}{l}
A_{1}=\frac{b_{3}}{2 \pi}, \\
A_{2}=\frac{\left(K_{2} \lambda_{11}+d_{15}^{2}\right) p-\left(R_{3} \lambda_{11}+e_{15} d_{15}\right) q-\left(K_{2} e_{15}-R_{3} d_{15}\right) v}{2 \pi \operatorname{det} \mathbf{C}} \\
B_{1}=\frac{b_{\perp}}{2 \pi}, \\
B_{2}=\frac{-\left(\lambda_{11} R_{3}+e_{15} d_{15}\right) p+\left(\lambda_{11} C_{44}+e_{15}^{2}\right) q-\left(C_{44} d_{15}-R_{3} e_{15}\right) v}{2 \pi \operatorname{det} \mathbf{C}} \\
C_{1}=\frac{b_{\varphi}}{2 \pi}, \\
C_{2}=\frac{\left(e_{15} K_{2}-R_{3} d_{15}\right) p+\left(C_{44} d_{15}-R_{3} e_{15}\right) q+\left(C_{44} K_{2}-R_{3}^{2}\right) v}{2 \pi \operatorname{det} \mathbf{C}} .
\end{array}\right.
$$

Introducing the generalized strains $\mathbf{Z}_{2}=\left[\varepsilon_{32}, \omega_{32},-E_{2}\right]$ and $\mathbf{Z}_{1}=\left[\varepsilon_{32}, \omega_{32},-E_{2}\right]$ and the generalized stresses $\Sigma_{2}=\left[\sigma_{32}, H_{32}, D_{2}\right]^{\mathrm{T}}$ and $\Sigma_{1}=\left[\sigma_{31}, H_{31}, D_{1}\right]^{\mathrm{T}}$, the generalized strain field and the stress field can be written as

$$
\begin{aligned}
& \mathrm{Z}_{2}+\mathrm{i} Z_{1}=\mathbf{f}_{0}^{\prime}(\zeta)=\frac{\mathbf{A}}{\zeta-\zeta_{d}}, \\
& \boldsymbol{\Sigma}_{2}+\mathrm{i} \boldsymbol{\Sigma}_{1}=\mathbf{C f}_{0}^{\prime}(\zeta)=\frac{\mathbf{C A}}{\zeta-\zeta_{d}} .
\end{aligned}
$$

According to equation (17), the generalized stress stimulated by dislocation $\zeta_{i}$ which is working on $\zeta_{1}$ is

$$
\mathbf{F}_{i 1}=\frac{\mathbf{C A}}{\zeta_{1}-\zeta_{i}}
$$

Now, we study the electroelastic solution of parallel screw dislocations. Taking the form of the corresponding matrix as $\mathbf{A}_{(i)}$, the generalized stress generated by dislocation $\zeta_{i}$ exerted on the arbitrary point $\zeta$ is

$$
\mathbf{F}_{0}(\zeta)=\sum_{i=1}^{n} \frac{\mathbf{C A}_{(i)}}{\zeta-\zeta_{i}}
$$

3.2. The Interaction of $n$ Parallel Screw Dislocations with Crack. It can be seen from the above discussion that the distribution force induced by dislocations on a semi-infinite crack sur- 
face is

$$
\mathbf{F}_{0}(\zeta)=\sum_{i=1}^{n} \frac{\mathbf{C A}(i)}{\zeta-\zeta_{i}}+\frac{\mathbf{C} \overline{\mathbf{A}}_{(i)}}{\zeta-\bar{\zeta}_{i}}, \quad-\infty<\xi_{1}<0, \xi_{2}=0 .
$$

Here, the condition of the free crack surface is considered; that is

$$
\operatorname{Re}\left[\mathbf{f}^{\prime}(\zeta)\right]^{\mathrm{T}}=0, \quad-\infty<\xi_{1}<0, \xi_{2}=0,
$$

where Re denotes the real part of an analytical function vector.

The physical meaning of the boundary condition equation (21) is that the additional distribution force $\mathbf{F}_{1}$ represents the cancelling force exerted on the boundary equal to the negative of $\mathbf{F}_{0}$. With the aid of equation (20), the boundary condition equation (21) leads to a system of the Riemann-Hilbert problem as

$$
\mathbf{F}_{1}^{+}\left(\xi_{1}\right)+\mathbf{F}_{1}^{-}\left(\xi_{1}\right)=-\mathbf{F}_{0}\left(\xi_{1}\right) .
$$

Using the standard methods given by Muskhelishvili [40], the general solution of equation (21) arrives at

$$
\mathbf{F}_{1}(\zeta)=-\frac{X(\zeta)}{2 \pi \mathrm{i}} \int_{-\infty}^{0} \frac{\mathbf{F}_{0}\left(\xi_{1}\right)}{X^{+}\left(\xi_{1}\right)\left(\xi_{1}-\zeta\right)} \mathbf{d} \xi_{1}+\mathbf{P}(\zeta) X(\zeta),
$$

where

$$
X(\zeta)=\zeta^{-(1 / 2)},
$$

and we have considered the single-valued condition of generalized displacements, i.e., $P(\zeta)=0$.

The solution of equation (20) together with equations (23) and (24) gives

$$
\mathbf{F}_{1}(\zeta)=-\sum_{i=1}^{n} \frac{\mathbf{C}}{2 \sqrt{\zeta}}\left(\frac{\mathbf{A}_{(i)}}{\sqrt{\zeta}+\sqrt{\zeta}}+\frac{\overline{\mathbf{A}}_{i}}{\sqrt{\zeta}+\sqrt{\bar{\zeta}_{i}}}\right) .
$$

Considering the stress field of the crack due to the farfield uniform loadings $\left(\sigma_{32}^{\infty}, H_{32}^{\infty}, E_{2}^{\infty}\right)$, equation (20) combined with equation (25) yields

$$
\mathbf{F}_{\text {total }}(\zeta)=\sum_{i=1}^{n} \frac{\mathbf{C}}{2 \sqrt{\zeta}}\left(\frac{\mathbf{A}_{(i)}}{\sqrt{\zeta}-\sqrt{\zeta} i}+\frac{\overline{\mathbf{A}}_{(i)}}{\sqrt{\zeta}-\sqrt{\bar{\zeta}}}\right)+\frac{\mathbf{C B}}{\sqrt{\zeta}},
$$

in which $\mathbf{B}=\left[A_{3}, B_{3}, C_{3}\right]^{\mathrm{T}}$ and

$$
\begin{aligned}
& A_{3}=\frac{\sigma_{32}^{\infty}-R_{3} \omega_{32}^{\infty}+e_{15} E_{2}^{\infty}}{C_{44}}, \\
& B_{3}=\omega_{32}^{\infty}, \\
& C_{3}=-E_{2}^{\infty} .
\end{aligned}
$$

Therefore, the analytic solution of electroelastic interaction of many parallel screw dislocations with the crack is

$$
\begin{aligned}
\mathbf{F}_{\text {total }}(z)= & \frac{\mathbf{F}_{\text {total }}(\zeta)}{m^{\prime}(\zeta)}=\sum_{i=1}^{n} \frac{\lambda \mathbf{C}}{2 z^{1-(\lambda / 2)}}\left(\frac{\mathbf{A}_{(i)}}{z^{\lambda / 2}-z_{i}^{\lambda / 2}}+\frac{\overline{\mathbf{A}}_{(i)}}{z^{\lambda / 2}-\bar{z}_{i}^{\lambda / 2}}\right) \\
& +\frac{\lambda \mathbf{C B}}{z^{1-(\lambda / 2)}} .
\end{aligned}
$$

If $\lambda=1$ and $i=1$, equation (28) reduces to the result in ref. [41] under some restrictions.

\section{Field Intensity Factors and Energy Release Rate}

4.1. Field Intensity Factors. In the $z$ plane, according to ref. [31], the phonon field stress, the phason field stress, and the electric displacement intensity factor at the wedge-shaped crack tip are defined as

$$
\mathbf{K}=\left[\begin{array}{c}
K_{\sigma_{32}}+\mathrm{i} K_{\sigma_{31}} \\
K_{H_{32}}+\mathrm{i} K_{H_{31}} \\
K_{D_{2}}+\mathrm{i} K_{D_{1}}
\end{array}\right]=\lim _{z \longrightarrow 0}(2 \pi z)^{1-(\lambda / 2)} \mathbf{F}_{\text {total }} .
$$

Actually, only $\varepsilon_{32}, \omega_{32}, E_{2}, \sigma_{32}, H_{32}$, and $D_{2}$ are singular at the crack tip. Substituting equation (28) to (29) and setting $z_{i}=r_{i} e^{i \theta_{i}}$, we obtain

$$
\begin{aligned}
K_{\sigma_{32}=} & \frac{\lambda}{\left(2 \pi r_{i}\right)^{\lambda / 2}} \sum_{i=1}^{n}\left[p \sin \frac{\lambda}{2} \theta_{i}-\left(C_{44} b_{3}+R_{3} b_{\perp}+e_{15} b_{\varphi}\right) \cos \frac{\lambda}{2} \theta_{i}\right]+\lambda(2 \pi)^{1-(\lambda / 2)} \sigma_{32}^{\infty}, \\
K_{H_{32}}= & \frac{\lambda}{\left(2 \pi r_{i}\right)^{\lambda / 2}} \sum_{i=1}^{n}\left[q \sin \frac{\lambda}{2} \theta_{i}-\left(R_{3} b_{3}+K_{2} b_{\perp}+d_{15} b_{\varphi}\right) \cos \frac{\lambda}{2} \theta_{i}\right] \\
& -\lambda(2 \pi)^{1-(\lambda / 2)}\left(d_{15} E_{2}^{\infty}-K_{2} \omega_{32}^{\infty}-\frac{R_{3}\left(e_{15} E_{2}^{\infty}-R_{3} \omega_{32}^{\infty}+\sigma_{32}^{\infty}\right)}{C_{44}}\right), \\
K_{D_{2}=} & -\frac{\lambda}{\left(2 \pi r_{i}\right)^{\lambda / 2}} \sum_{i=1}^{n}\left[v \sin \frac{\lambda}{2} \theta_{i}+\left(e_{15} b_{3}+d_{15} b_{\perp}-\lambda_{11} b_{\varphi}\right) \cos \frac{\lambda}{2} \theta_{i}\right] \\
& +\lambda(2 \pi)^{1-(\lambda / 2)}\left(\lambda_{11} E_{2}^{\infty}+d_{15} \omega_{32}^{\infty}+\frac{e_{15}\left(e_{15} E_{2}^{\infty}-R_{3} \omega_{32}^{\infty}+\sigma_{32}^{\infty}\right)}{C_{44}}\right) .
\end{aligned}
$$


Equations (30)-(32) agree with Chen's results [31] if there is only one dislocation and the phason field and the applied generalized stress are not considered. If the applied loading is $\left(\sigma_{32}^{\infty}, H_{32}^{\infty}, E_{2}^{\infty}\right)$ and the matrix only contains one dislocation whose core are not under the line force, the above equations can reduce to Li's results [3]. On the basis of the above restrictions, if the phason field is neglected, the solutions agree with those in ref. [34]. Moreover, equations (30)-(32) suggest that $K_{\sigma_{32}}$ is independent of $q$ and $v, K_{H_{32}}$ is independent of $p$ and $v$, and $K_{D_{2}}$ is independent of $p$ and $q$, if all $b_{3}, b_{\perp}, b_{\varphi}, p, q$, and $v$ are supposed to be positive.

4.2. Energy Release Rate. The energy release rate can be calculated as [42]

$$
G=\frac{1}{4} \mathbf{K}^{\mathrm{T}} \mathbf{H K}
$$

where $\mathbf{H}=2 \mathbf{C}^{-1}$.

Thus, the energy release rate for the interaction of the wedge-shaped crack with screw dislocations is derived as

$$
\begin{aligned}
G= & \frac{1}{2 \operatorname{det} C}\left[\left(-d_{15}^{2}-K_{2} \lambda_{11}\right) K_{\sigma_{32}}^{2}+\left(-e_{15}^{2}-C_{44} \lambda_{11}\right) K_{H_{32}}^{2}\right. \\
& +\left(C_{44} K_{2}-R_{3}^{2}\right) K_{D_{2}}^{2}+2\left(d_{15} e_{15}+R_{3} \lambda_{11}\right) K_{\sigma_{32}} K_{H_{32}} \\
& \left.+2\left(-C_{44} d_{15}+e_{15} R_{3}\right) K_{H_{32}} K_{D_{2}}+2\left(-e_{15} K_{2}+d_{15} R_{3}\right) K_{\sigma_{32}} K_{D_{2}}\right] .
\end{aligned}
$$

\section{Image Force}

The image force is expressed as [43]

$$
\left\{\begin{array}{l}
F_{1}=b_{3} \sigma_{32}^{\mathrm{T}}+b_{\perp} H_{32}^{\mathrm{T}}+b_{\varphi} D_{2}^{\mathrm{T}}+p \varepsilon_{31}^{\mathrm{T}}+q \omega_{31}^{\mathrm{T}}+v E_{1}^{\mathrm{T}}, \\
F_{2}=-b_{3} \sigma_{31}^{\mathrm{T}}-b_{\perp} H_{31}^{\mathrm{T}}-b_{\varphi} D_{1}^{\mathrm{T}}+p \varepsilon_{32}^{\mathrm{T}}+q \omega_{32}^{\mathrm{T}}+v E_{2}^{\mathrm{T}},
\end{array}\right.
$$

where stresses and strains with superscript " $T$ " are the perturbation stresses and strains at the dislocation point.

Therefore, the image force exerted on the dislocation at $z_{d}\left(z_{d}\right.$ is excluded from $\left.z_{i}\right)$ in the presence of a wedge-shaped crack and a collection of other dislocations at $z_{i}$ can be expressed as

$$
\begin{aligned}
& \bar{f}_{d}=F_{1}-\mathrm{i} F_{2}=\frac{\mathbf{b C}}{m^{\prime}\left(\zeta_{d}\right)}\left[-\frac{\mathbf{A}}{4 \zeta_{d}}+\frac{\overline{\mathbf{A}}}{2 \sqrt{\zeta_{d}}\left(\sqrt{\zeta_{d}}-\sqrt{\bar{\zeta}_{d}}\right)}\right] \\
&+\frac{\mathrm{i} \mathbf{b}^{\prime}}{m^{\prime}\left(\zeta_{d}\right)}\left[-\frac{\mathbf{A}}{4 \zeta_{d}}+\frac{\overline{\mathbf{A}}}{2 \sqrt{\zeta_{d}}\left(\sqrt{\zeta_{d}}-\sqrt{\zeta_{d}}\right)}\right]+ \\
& \sum_{i}\left\{\frac{\mathbf{b C}}{m^{\prime}\left(\zeta_{d}\right)}\left[\frac{\mathbf{A}_{(i)}}{2 \sqrt{\zeta_{d}}\left(\sqrt{\zeta_{d}}-\sqrt{\zeta_{i}}\right)}+\frac{\overline{\mathbf{A}}_{(i)}}{2 \sqrt{\zeta_{d}}\left(\sqrt{\zeta_{d}}-\sqrt{\zeta_{i}}\right)}\right]\right. \\
&\left.+\frac{\mathbf{i \mathbf { b } ^ { \prime }}}{m^{\prime}\left(\zeta_{d}\right)}\left[\frac{\mathbf{A}_{(i)}}{2 \sqrt{\zeta_{d}}\left(\sqrt{\zeta_{d}}-\sqrt{\zeta_{i}}\right)}+\frac{\overline{\mathbf{A}}_{(i)}}{2 \sqrt{\zeta_{d}}\left(\sqrt{\zeta_{d}}-\sqrt{\zeta_{i}}\right)}\right]\right\} \\
&+\frac{\mathbf{b C B}+\mathbf{i} \mathbf{b}^{\prime} \mathbf{B}}{m^{\prime}\left(\zeta_{d}\right) \sqrt{\zeta_{d}}}
\end{aligned}
$$


TABLe 1: Material constants in the 1D hexagonal piezoelectric quasicrystal.

\begin{tabular}{lccccc}
\hline$C_{44}\left(\mathrm{Nm}^{-2}\right)$ & $R_{3}\left(\mathrm{Nm}^{-2}\right)$ & $K_{2}\left(\mathrm{Nm}^{-2}\right)$ & $e_{15}\left(\mathrm{Cm}^{-2}\right)$ & $d_{15}\left(\mathrm{Cm}^{-2}\right)$ & $\lambda_{11}\left(C^{2} N^{-1} m^{-2}\right)$ \\
\hline $5 \times 10^{10}$ & $1.20 \times 10^{9}$ & $0.30 \times 10^{9}$ & -0.138 & -0.16 & $82.6 \times 10^{-12}$ \\
\hline
\end{tabular}

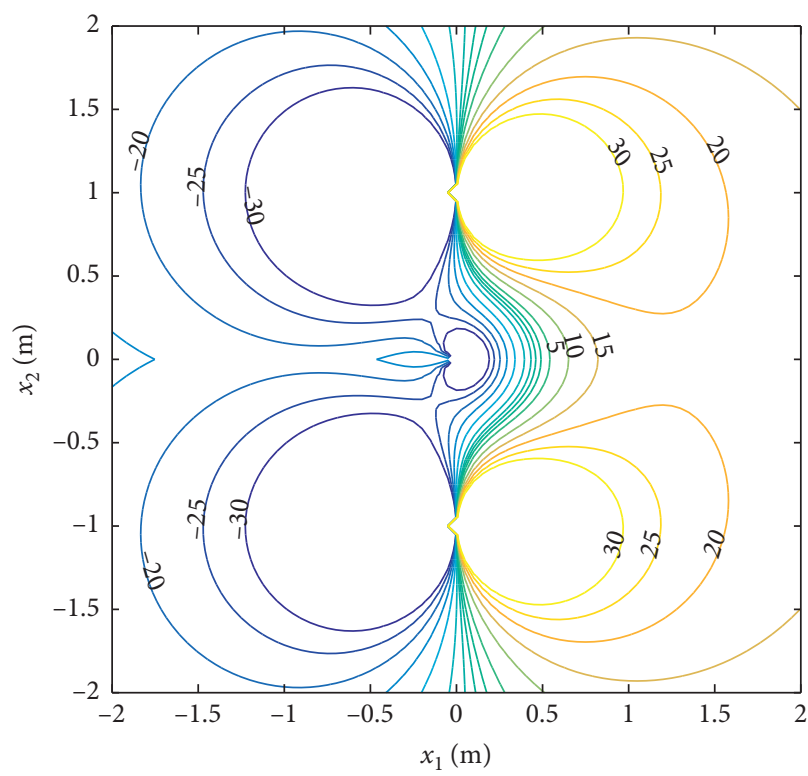

(a)

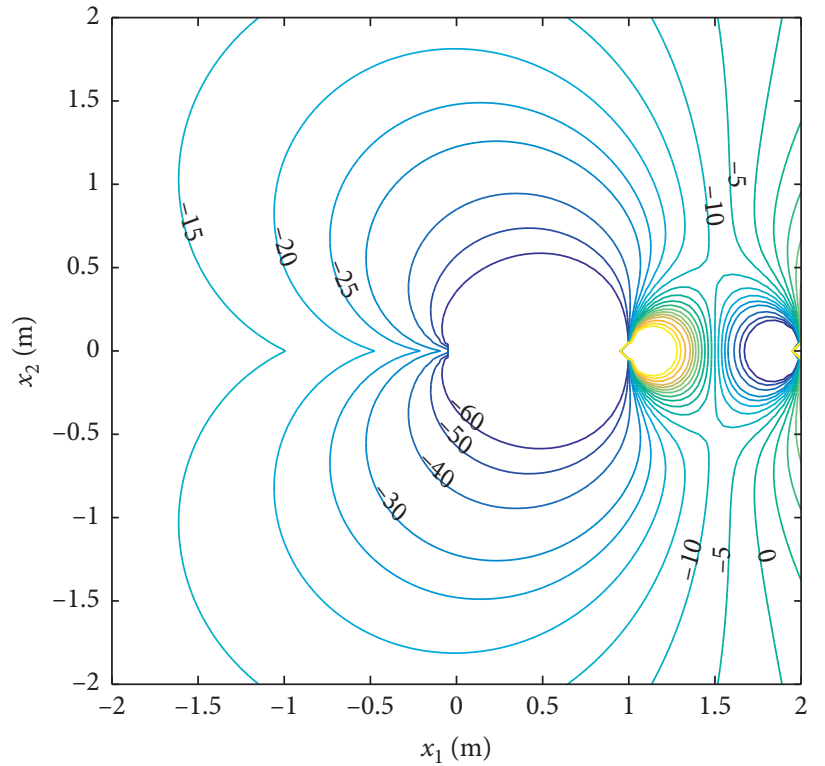

(c)

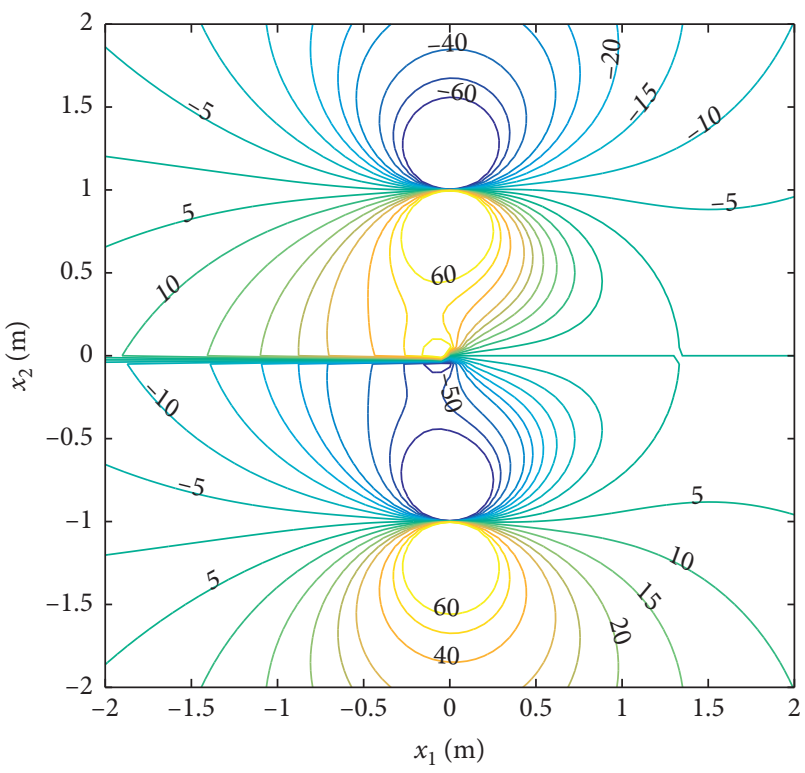

(b)

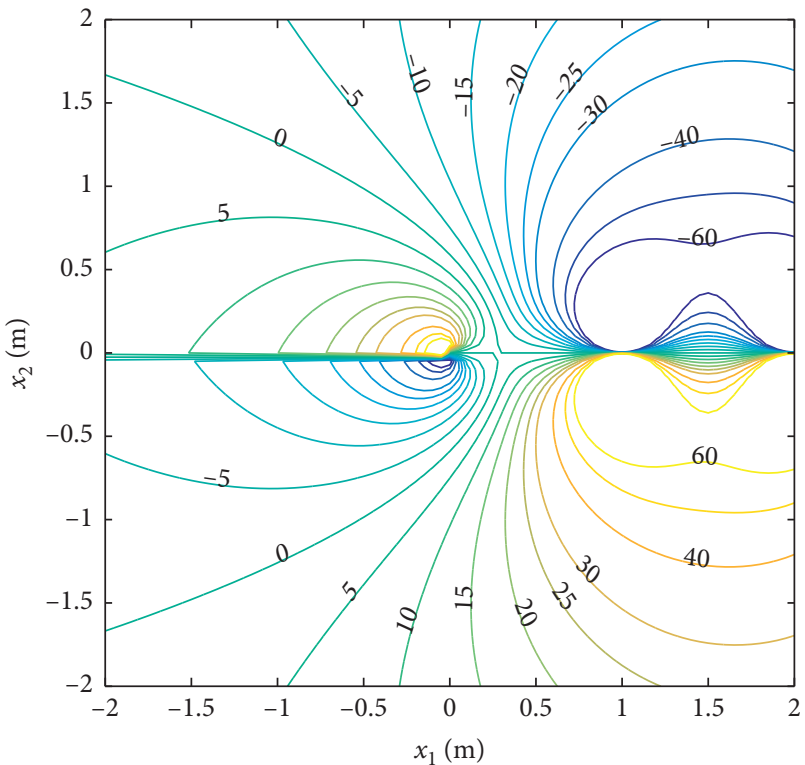

(d)

FIgURe 3: Contours of phonon field stress components are induced by dislocations situated at $(0,1)$ and $(0,-1)$, (a) $\sigma_{32}$ and (b) $\sigma_{31}$, and at $(1,0)$ and $(2,0)$, (c) $\sigma_{32}$ and (d) $\sigma_{31}$.

where

$$
\begin{aligned}
\mathbf{b} & =\left(b_{3}, b_{\perp}, b_{\varphi}\right), \\
\mathbf{b}^{\prime} & =(p, q, v) .
\end{aligned}
$$

Noting that the above solution can be reduced to Lin and Thomson [35] in the case of $\lambda=1$ and the material is pure elastic. Furthermore, equation (36) is comprised of three terms. The first term containing the quadratic of $\left(b, b^{\prime}\right)$ coming from the image dislocation. The second term containing $\left(b, b^{\prime}\right)$ and $\left(b^{(i)}, b^{\prime(i)}\right)$ which are the interaction forces from the other dislocations acting on the dislocation $z_{d}$. The last one containing $\left(b, b^{\prime}\right)$ which is induced by the applied generalized stress. 


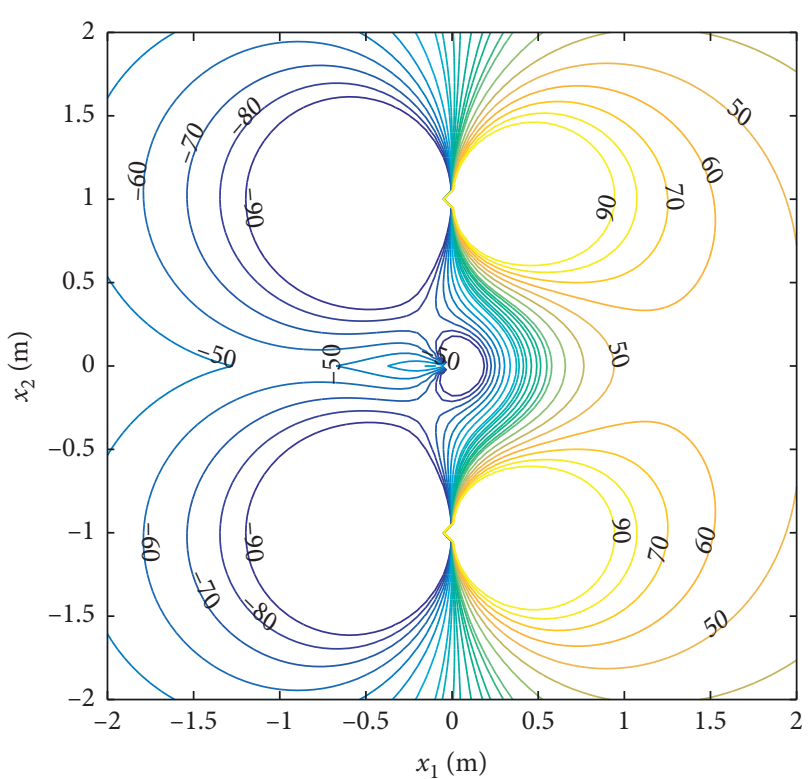

(a)

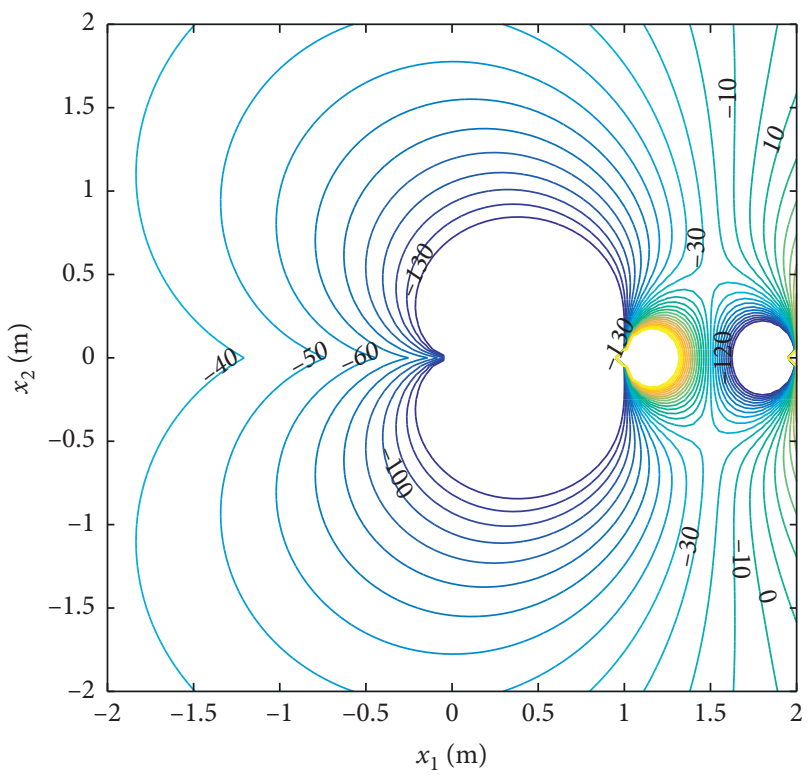

(c)

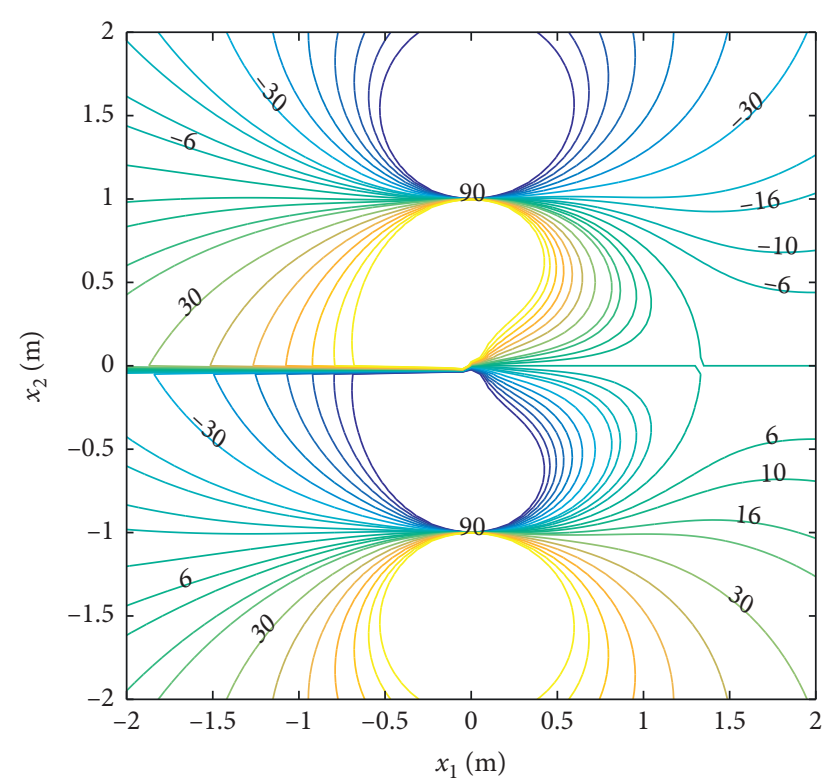

(b)

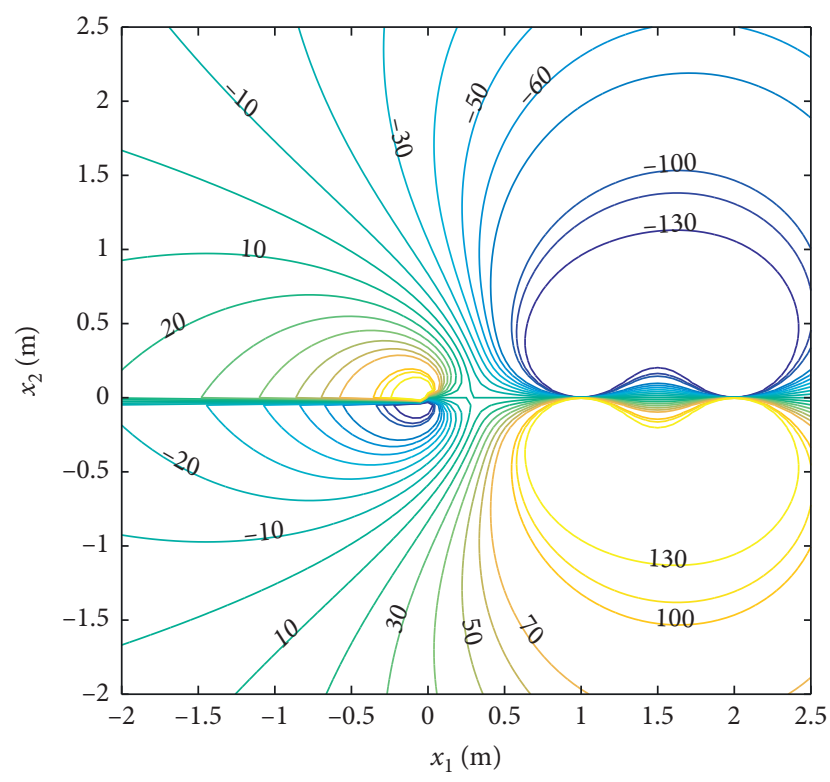

(d)

FIGURE 4: Contours of phason field stress components are induced by dislocations situated at $(0,1)$ and $(0,-1),(\mathrm{a}) H_{32} / 10^{-2}$ and $(\mathrm{b})$ $H_{31} / 10^{-2}$, and at $(1,0)$ and $(2,0),\left(\right.$ c) $H_{32} / 10^{-2}$ and (d) $H_{31} / 10^{-2}$.

When all the dislocations are located at the real axis, the solution can be simplified as

$$
\begin{aligned}
\bar{f}_{d} & =-\frac{\mathbf{b C A}}{4 m^{\prime}\left(\xi_{d}\right) \xi_{d}}-\frac{i \mathbf{b}^{\prime} \mathbf{A}}{4 m^{\prime}\left(\xi_{d}\right) \xi_{d}} \\
& +\sum_{i}\left\{\frac{\mathbf{b C A}_{(i)}}{m^{\prime}\left(\xi_{d}\right) \sqrt{\xi_{d}}\left(\sqrt{\xi_{d}}-\sqrt{\xi_{i}}\right)}+\frac{i \mathbf{b}^{\prime} \mathbf{A}_{(i)}}{m^{\prime}\left(\xi_{d}\right) \sqrt{\xi_{d}}\left(\sqrt{\xi_{d}}-\sqrt{\xi_{i}}\right)}\right\} \\
& +\frac{\mathbf{b C B}+\mathbf{i b}^{\prime} \mathbf{B}}{m^{\prime}\left(\xi_{d}\right) \sqrt{\xi_{d}}}
\end{aligned}
$$

i.e.,

$$
\begin{aligned}
\bar{f}_{d}= & -\frac{\mathbf{b} \mathbf{C A}}{4 x_{d}}-\frac{i \mathbf{b}^{\prime} \mathbf{A}}{4 x_{d}}+\sum_{i} \frac{\mathbf{b C A}_{(i)}}{x_{d}^{1-(\lambda / 2)}\left(x_{d}^{\lambda / 2}-x_{i}^{\lambda / 2}\right)} \\
& +\frac{i \mathbf{b}^{\prime} \mathbf{A}_{(i)}}{x_{d}^{1-(\lambda / 2)}\left(x_{d}^{\lambda / 2}-x_{i}^{\lambda / 2}\right)}+\frac{\mathbf{b} \mathbf{C} \mathbf{B}+i \mathbf{b}^{\prime} \mathbf{B}}{x_{d}^{1-(\lambda / 2)}} .
\end{aligned}
$$

\section{Numerical Example}

Although the field intensity factors, energy release rate, and the image force acting on the dislocation are expressed 


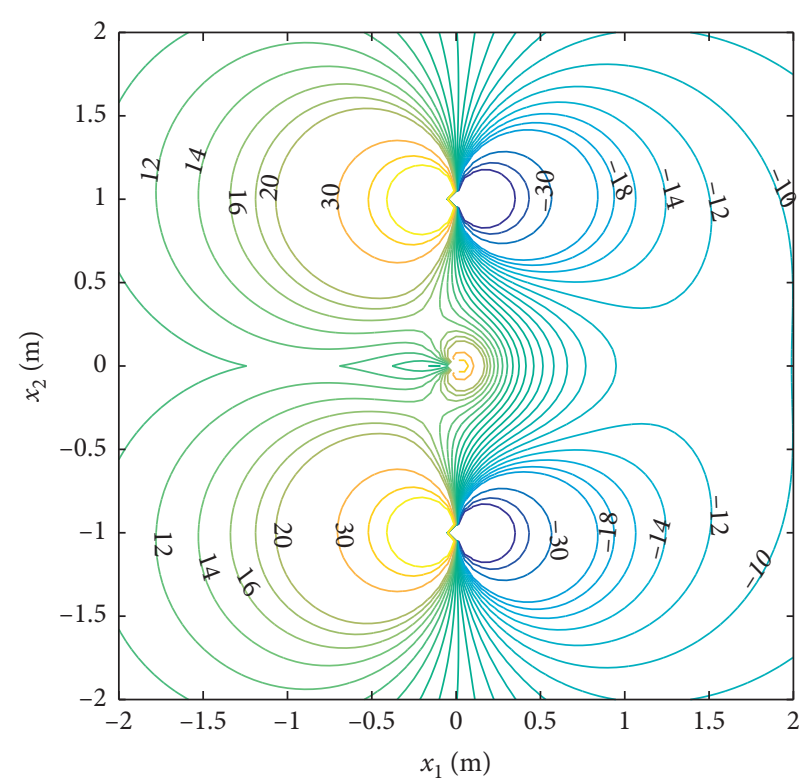

(a)

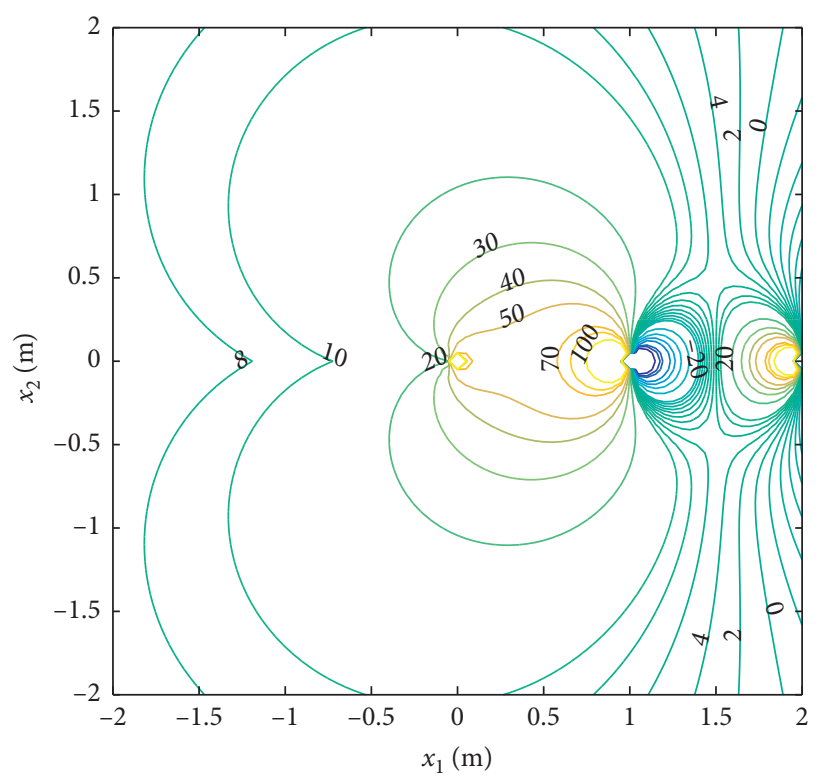

(c)

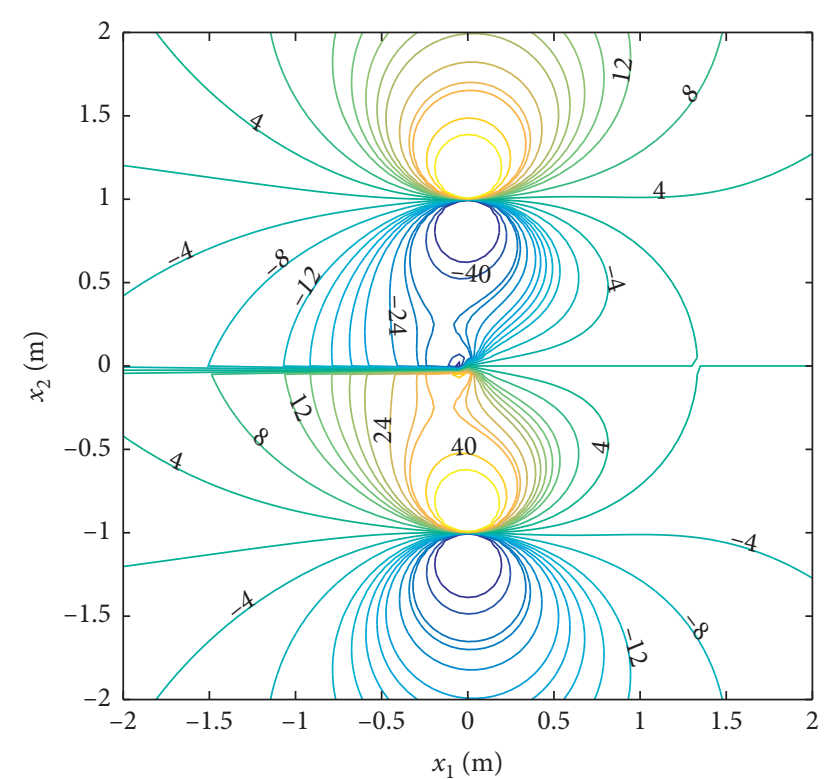

(b)

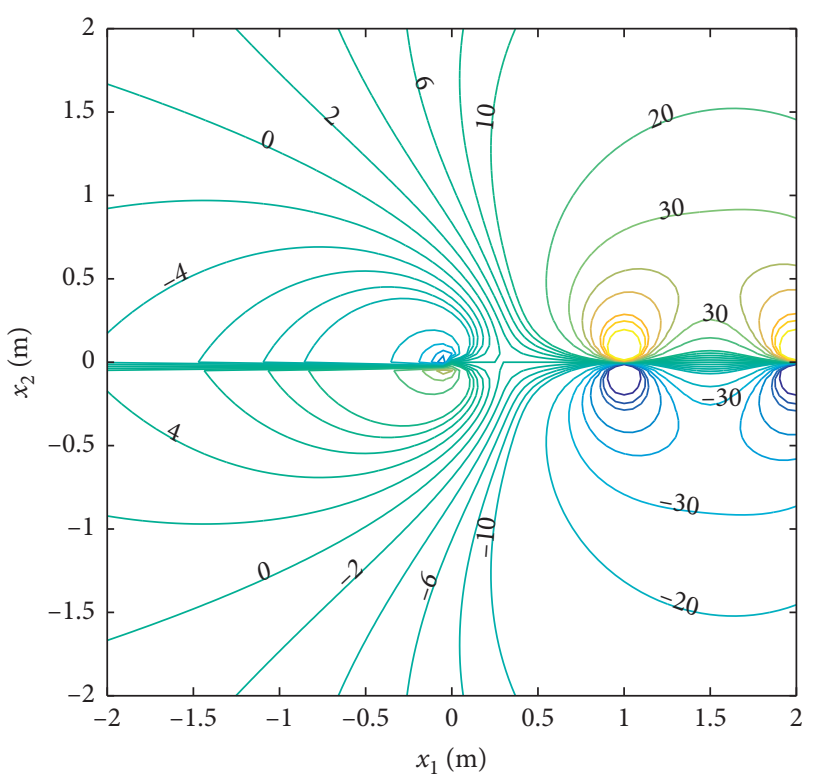

(d)

FIGURE 5: Contours of electric displacement components are induced by dislocations situated at $(0,1)$ and $(0,-1),(a) D_{2} / 10^{-11}$ and $(b)$ $D_{1} / 10^{-11}$, and at $(1,0)$ and $(2,0),\left(\right.$ c) $D_{2} / 10^{-12}$ and (d) $D_{1} / 10^{-12}$.

explicitly in the previous sections, it is not straightforward that how the dislocation location, dislocation distribution, the wedge angle, and the number of dislocations affect them. The numerical examples are therefore performed in this section. The material constants we choose are listed in Table 1 [14].

The Burgers vectors, line forces, and line charge at the dislocation core are

$$
\begin{aligned}
& b_{3}=b_{\perp}=1.0 \times 10^{-9} \mathrm{~m}, \\
& b_{\varphi}=1.0 \times 10^{-9} \mathrm{~V}, \\
& p=q=10 \mathrm{~N} / \mathrm{m}, \\
& v=1.0 \times 10^{-3} \mathrm{C} / \mathrm{m} .
\end{aligned}
$$




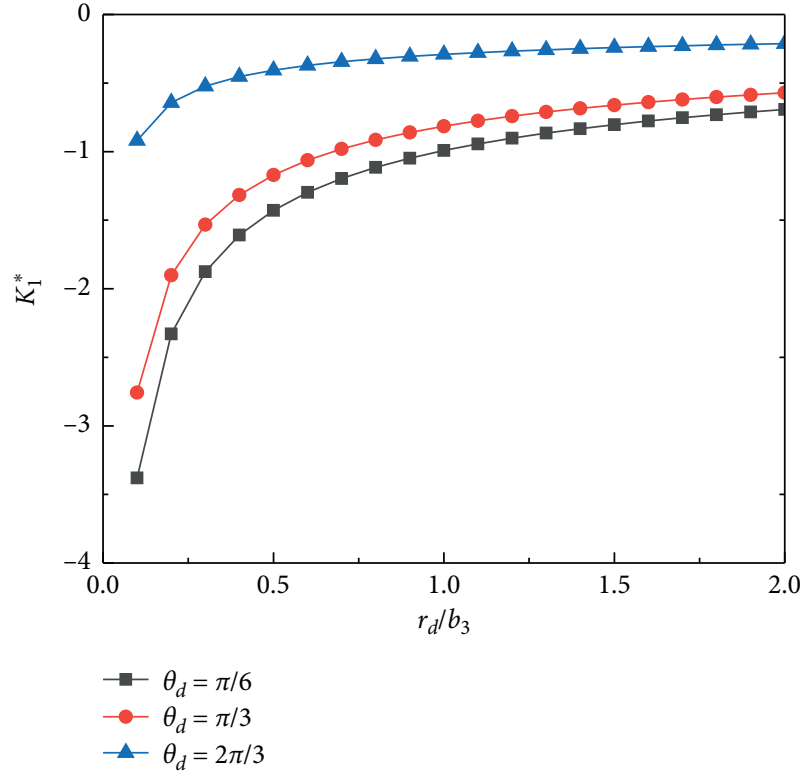

(a)

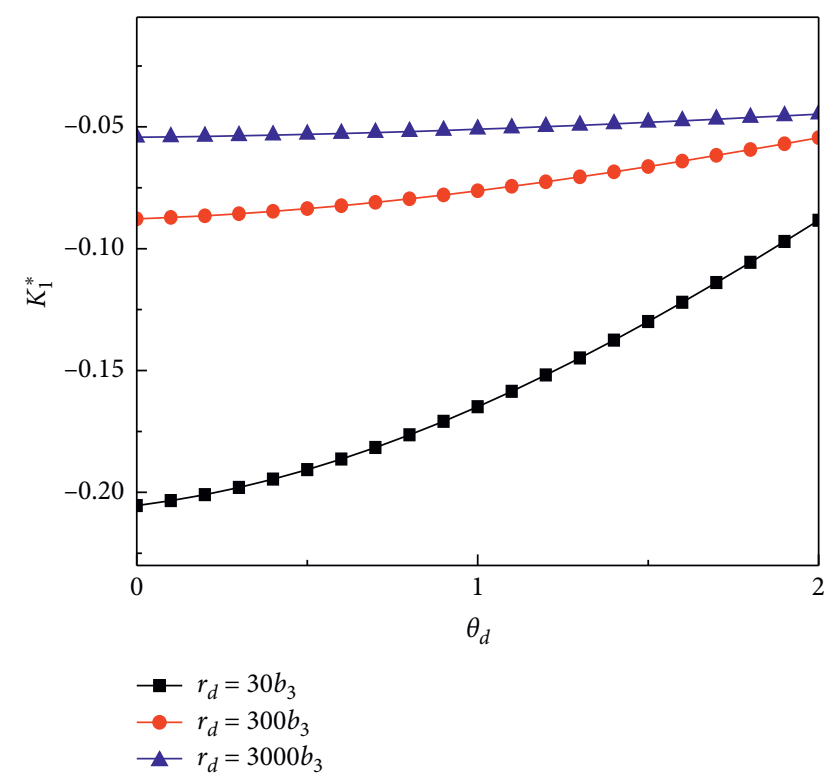

(b)

Figure 6: (a) $K_{1}^{*}$ varies with $r_{d} / b_{3}$. (b) $K_{1}^{*}$ varies with $\theta_{d}$.

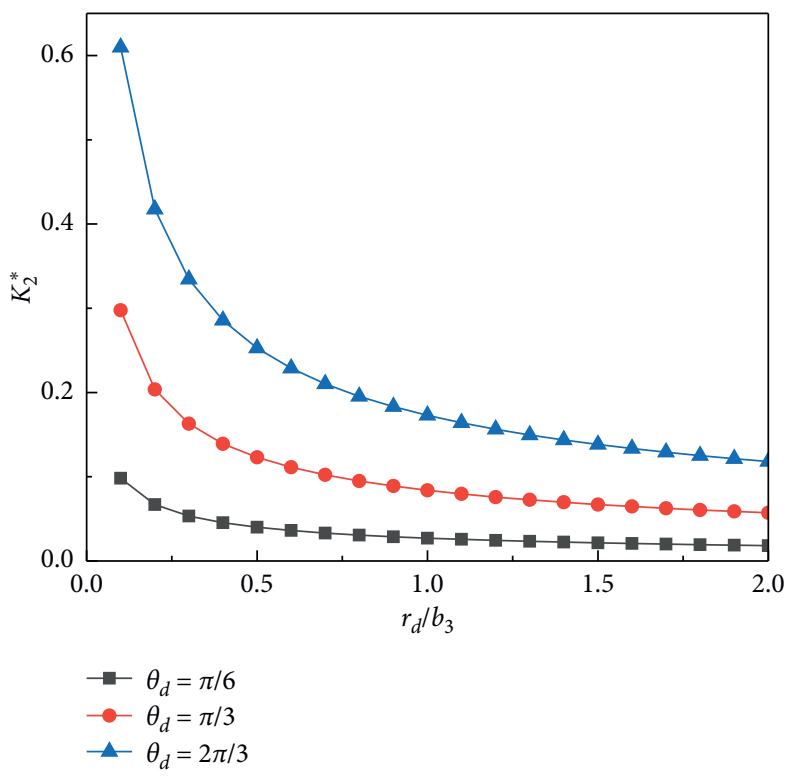

(a)

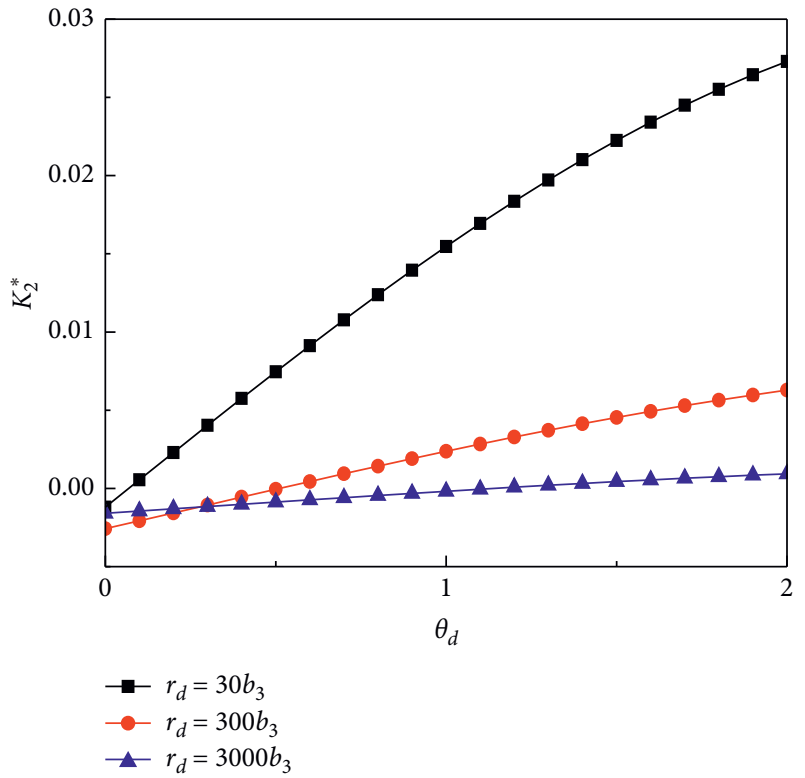

(b)

Figure 7: (a) $K_{2}^{*}$ varies with $r_{d} / b_{3}$. (b) $K_{2}^{*}$ varies with $\theta_{d}$.

The critical energy release rate is taken as $G_{r}=5 \mathrm{~N} / \mathrm{m}$ and the wedge angle in the following numerical discussions is $\pi / 6$. For the sake of discussion, the magnitude of all Burgers vectors is regarded as $10^{-9}$ and all the dislocation have the same value of $(p, q, v)$.

Figures $3-5$, respectively, show the phonon field stress, phason field stress, and electric displacement component contours due to the dislocations. When the dislocations are located at the points $(0,1)$ and $(0,-1)$, one may note that as the lines of $\sigma_{32}, H_{32}$, and $D_{2}$ get closer to the crack surface, they become parallel to the surface and the generalized stress becomes smaller. The phonon field stress $\sigma_{32}$ vanishes when it reaches the crack surface, as well as the phason field stress $H_{32}$ and electric displacement $D_{2}$, so that the boundary condition is satisfied. The lines of $\sigma_{31}, H_{31}$, and $D_{1}$ are antisymmetric about the straight line $x_{2}=0$, but the lines of $\sigma_{32}, H_{32}$, and $D_{2}$ are symmetric about the straight line $x_{2}=0$. These results also reveal that the phonon field stress, 


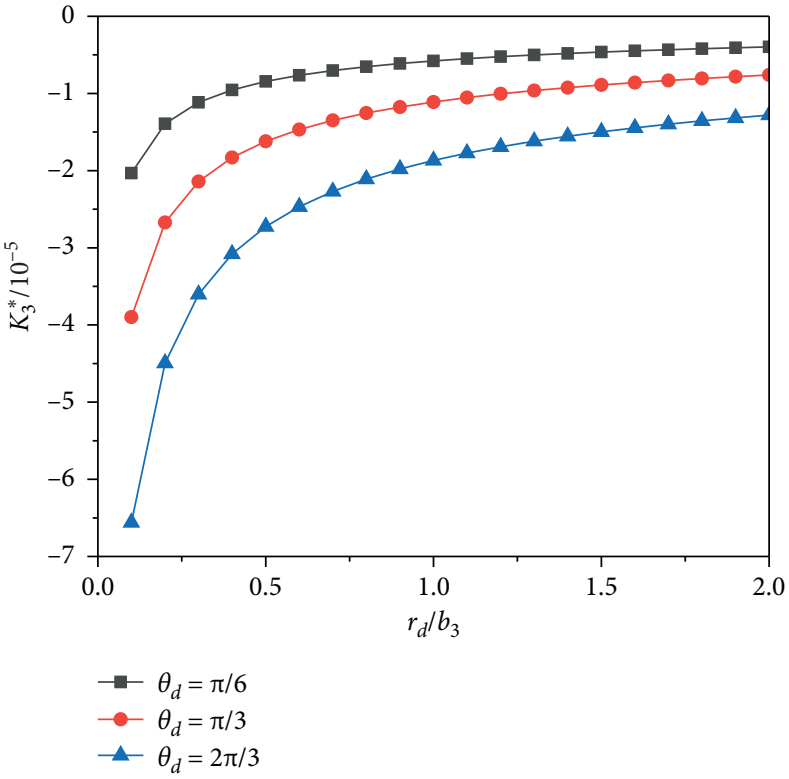

(a)

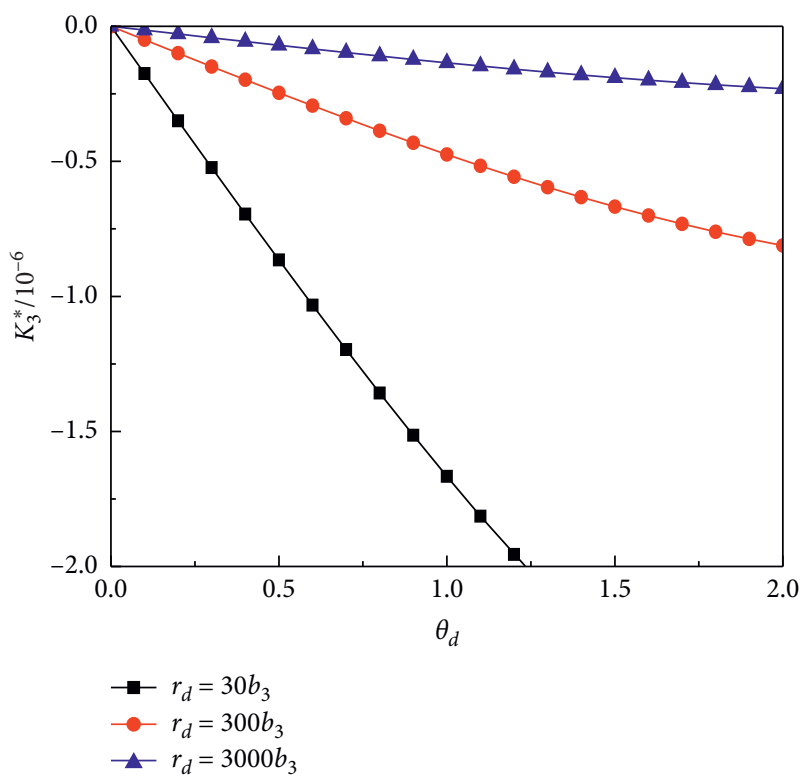

(b)

FiguRE 8: (a) $K_{3}^{*} / 10^{-5}$ varies with $r_{d} / b_{3}$. (b) $K_{3}^{*} / 10^{-6}$ varies with $\theta_{d}$.

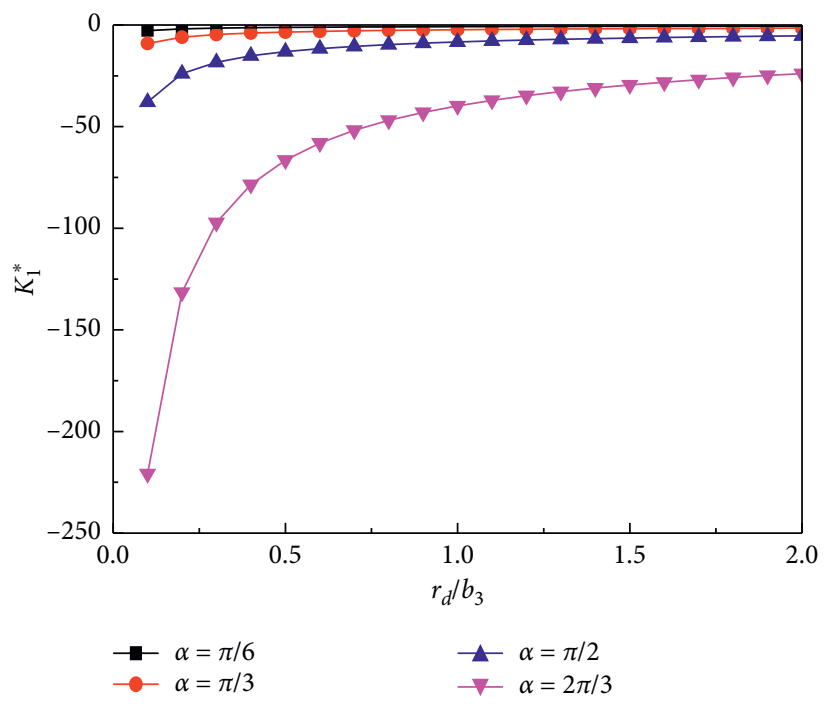

(a)

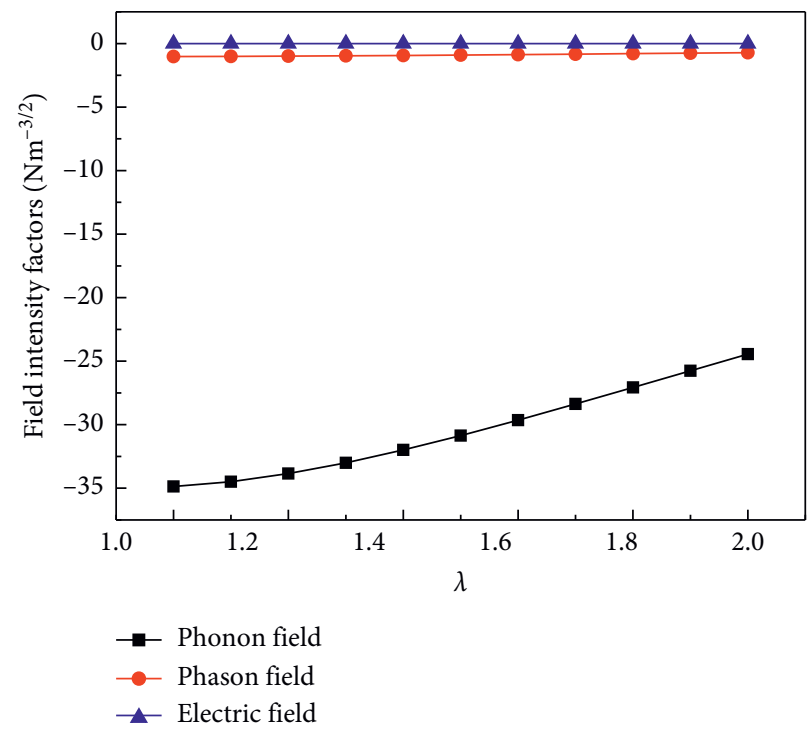

(b)

Figure 9: (a) $K_{1}^{*}$ varies with $r_{d} / b_{3}$ of different $\alpha$. (b) Field intensity factors vary with $\lambda$.

phason field stress, and the electric displacement field show square root singularity at the crack tip while having one order singularity at the location of dislocation. The phonon field stress contours have the same trend as the phason field stress contours but is opposite to the electric field. In addition, the magnitude of the electric displacement is less than the magnitude of the phason field stress, but the magnitude of the phason field stress is less than the magnitude of the phonon field stress.
Supposing there are already two dislocations located at $x_{1}=1000 \times b_{3}$ and $x_{2}=2000 \times b_{3}$, respectively, the relationship between the field intensity factors and the positions of the other dislocation is exhibited in Figures 6-8 without remote uniform loading. Let us define the normalized phonon field intensity factor, phason field intensity factor, and electric displacement intensity factor as $K_{1}^{*}=K_{\sigma_{32}} / C_{44} \sqrt{b_{3}}, \quad K_{2}^{*}=K_{H_{32}} / C_{44} \sqrt{b_{3}}, \quad$ and $K_{3}^{*}=K_{D_{2}} / C_{44} \sqrt{b_{3}}$, respectively. It can be seen that the value 


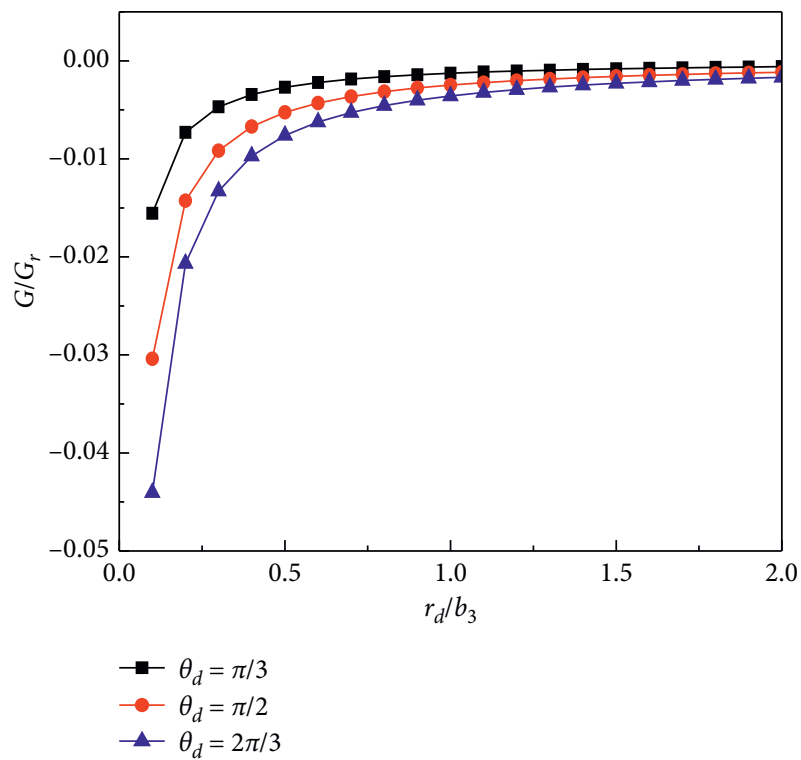

(a)

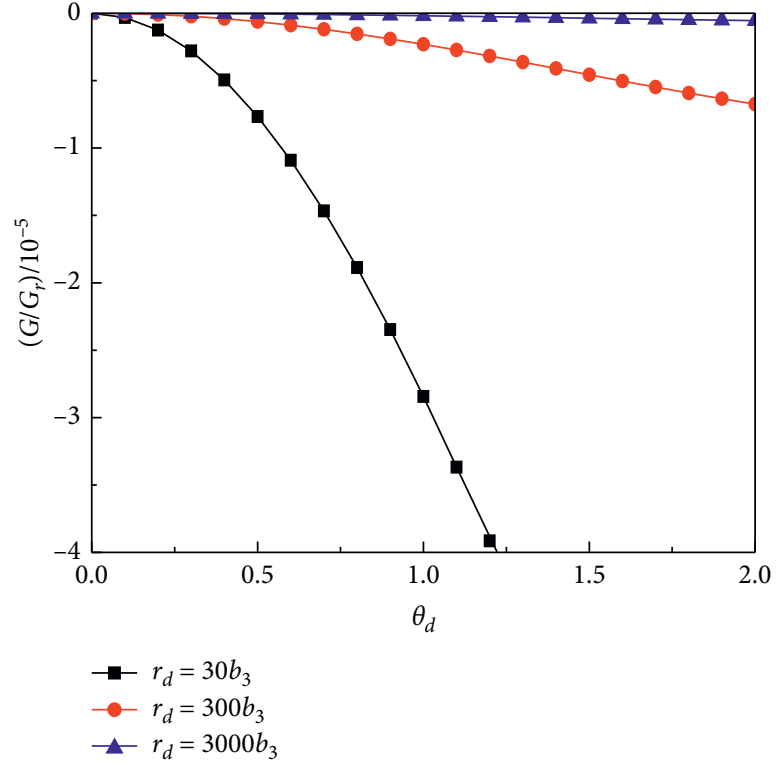

(b)

Figure 10: (a) $G / G_{r}$ varies with $r_{d} / b_{3}$. (b) $\left(G / G_{r}\right) / 10^{-5}$ varies with $\theta_{d}$.

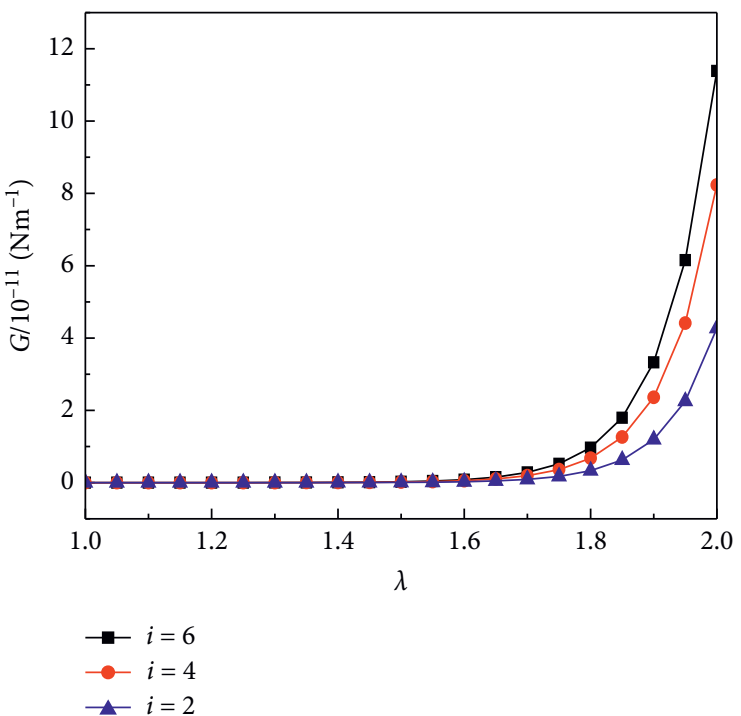

(a)

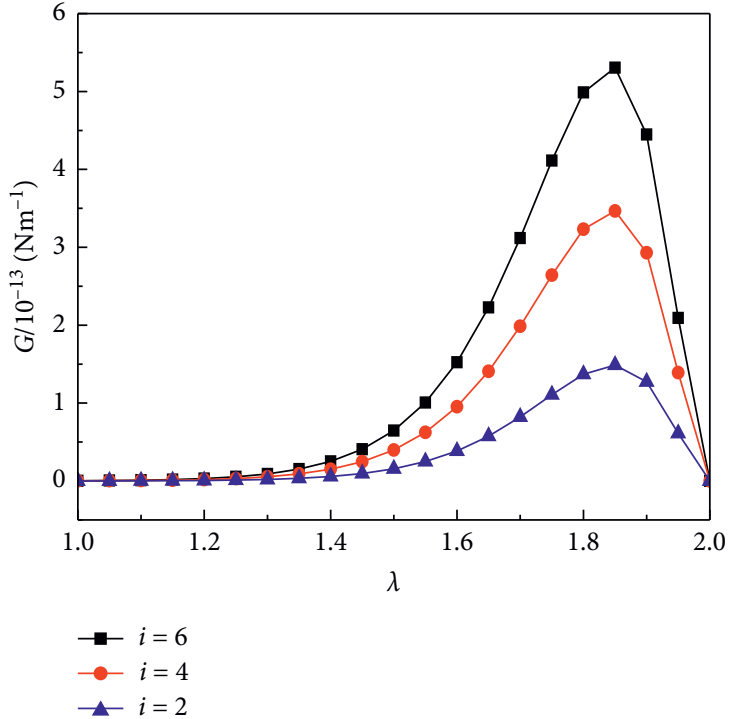

(b)

FIGURE 11: (a) Energy release rate varies with $\lambda$ when all the dislocations are located at the real axis. (b) Energy release rate varies with $\lambda$ when all the dislocations are located at the imaginary axis symmetrically.

of $K_{1}^{*}$ and $K_{3}^{*}$ is always negative, but the value of $K_{2}^{*}$ is always positive. We find that the effects of the dislocation position on the three intensity factors are different. Figures 6(a) and Figure 6(b) show that the shielding effect in the phonon field quickly decreases with the value of the normalized distance $r_{d} / b_{3}$ increasing, while decreases with the value of $\theta_{d}$ increasing. From Figures 7(a) and Figure 7(b), it is interesting to note that $K_{2}^{*}$ increases with the increasing $\theta_{d}$, while decreases with the increasing $r_{d} / b_{3}$. When the dislocation is located at the real axis, the stress intensity factor is positive.
Figures 8(a) and Figure 8(b) indicate that the shielding effect on the electric field decreases as $r_{d} / b_{3}$ increases, while it decreases as $\theta_{d}$ decreases. Taking the stress intensity factor of the phonon field as an example, Figure 9(a) illustrates that $K_{1}^{*}$ increases with $\alpha$, and the larger the wedge angle is, the stronger the shielding effect will become. The variations in the three field intensity factors with the wedge angle are plotted in Figure 9(b). When the two dislocations are located at $x_{1}=1$ and $x_{2}=2$, it can be found that the phonon field stress intensity factor increases as the wedge angle increases, 


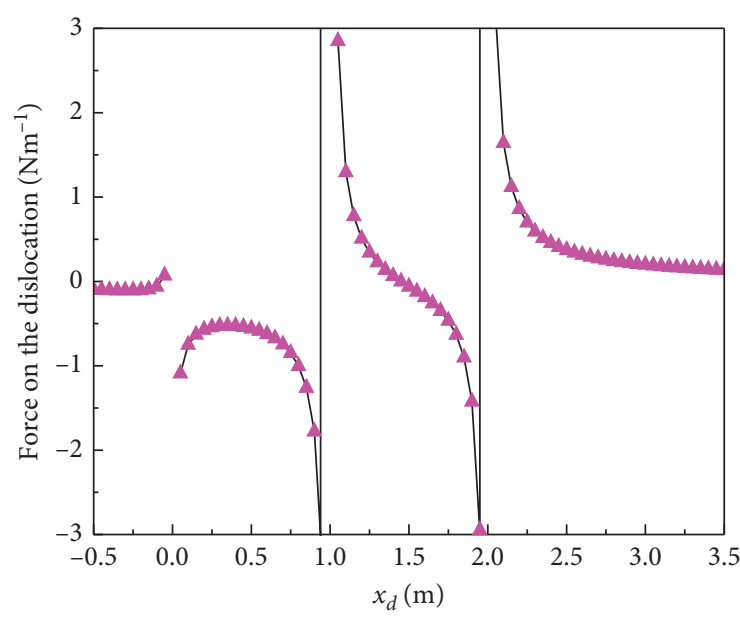

(a)

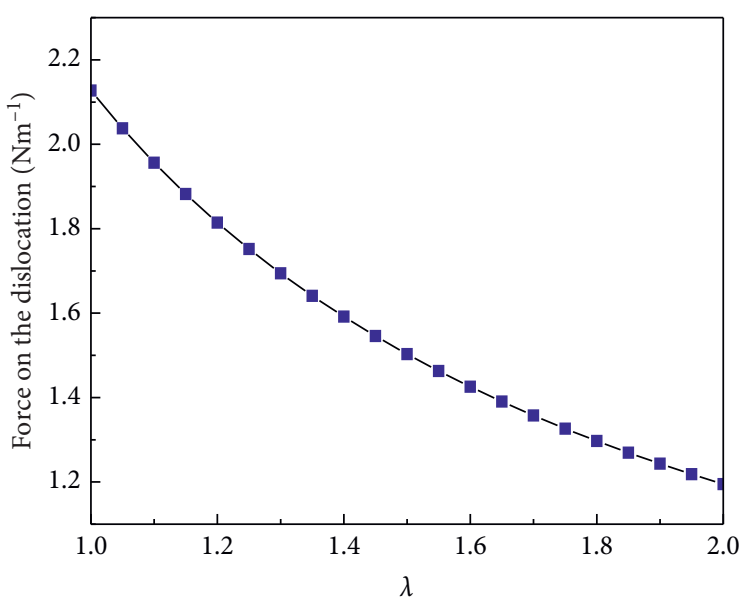

(b)

Figure 12: (a) The force acting on the dislocation $x_{d}$ varies with the distance from the dislocation $x_{d}$ to the crack tip when the other dislocations are located at $(1,0)$ and $(2,0)$. (b) The force acting on the dislocation $x_{d}$ varies with $\lambda$ when the other dislocations are located at $(1,0)$ and $(2,0)$.

but $\lambda$ has little effect on the phason field stress intensity factor and the electric displacement intensity factor since the material constants related to the phonon field are far greater than those related to the phason field and the electric field.

Similarity, there exist two dislocations located at $x_{1}=$ $1000 \times b_{3}$ and $x_{2}=2000 \times b_{3}$, respectively. In this case, Figures 10(a) and 10(b) more clearly show the retarding effects, respectively, of $r_{d} / b_{3}$ and $\theta_{d}$ on the energy release rate without far-field loading. The normalized energy release rate $G / G_{r}$ increases as $d \theta$ decreases. In contrast, $G / G_{r}$ increases as $r_{d} / b_{3}$ increases. Figure 10(a) implies that $r_{d} / b_{3}$ contributes none to the energy release rate when its value increases to a certain extent. Figure 10(b) illustrates that $r_{d} / b_{3}$ does not affect the energy rate when all the dislocations are situated at the real axis. Figures 11(a) and Figure 11(b) depict the effect of the wedge angle on the energy release rate when the dislocations are in different positions. The energy release rate is positive whether the dislocations are located at the real axis or symmetrically distributed at the imaginary axis, which means the wedge angle accelerates the crack propagation. $G$ increases as the number of dislocations increases. Also, $G$ increases with the increasing $\lambda$ when all the dislocations are located at the real axis, while it increases first and then decreases when the dislocations are situated at the imaginary axis symmetrically.

Making all dislocations located at the real axis, and $x_{1}=$ 1 and $x_{2}=2$, in the absence of the remote applied generalized stress, Figure 12(a) shows that $\bar{f}_{d}$ is always negative when the dislocation $x_{d}$ is between the crack tip and dislocation $x_{1}$, which means dislocation $x_{d}$ is always attracted. When the dislocation $x_{d}$ from $x_{2}$ moves to $x_{1}$, it is first rejected and then attracted, and the attractive force is gradually increased and the repulsive force is gradually reduced. When the dislocation $x_{d}$ is farther away from $x_{2}$, it is always rejected and the repulsive force is reduced. Figure 12(b) implies that the force on the dislocation is always positive and the magnitude decreases when increasing the wedge angle.

\section{Conclusions}

In this paper, the fracture mechanic model of the two-dimensional crack and parallel dislocations is established. The interaction between parallel screw dislocations and a wedgeshaped crack in a one-dimensional hexagonal quasicrystal with piezoelectric effects is studied, and the closed-form solutions of the electroelastic field, field intensity factors, energy release rate, and generalized Peach-Koehler force are obtained. The analytical solutions can reduce to the results from the existing literature. The disadvantage of the present solution is that the dislocations are considered to distribute uniformly in the numerical examples which do not reflect the microstructure of the material. The solutions are limited in numbers of dislocations, and only the same sign dislocations are considered here. From the numerical illustrations, some important results are summarized in the following outline:

(1) The trend of contours of the phonon field stress is similar to the phason field stress but is different from the electric displacement.

(2) The stress concentration occurs at the crack tip and dislocations cores. The effects of the location of dislocations on the three field intensity factors are different, and the wedge angle always increases the shielding effect.

(3) The increasing number of dislocations always accelerates the crack propagation. The wedge angle accelerates the crack growth when dislocations are located at the real axis, but it first promotes crack growth and then demotes crack propagation when dislocations are located at the imaginary axis symmetrically.

(4) The image force acting on the dislocation in the presence of a crack and other dislocations decreases when increasing the wedge angle. The dislocation is 
either attracted or rejected in the process of moving from left to right along the real axis.

\section{Data Availability}

The data used to support the findings of this study are included within the article.

\section{Conflicts of Interest}

The authors declare that they have no conflicts of interest.

\section{Acknowledgments}

This project was supported by the National Natural Key R\&D Program of China (Grant no. 2017YFC1405605) and the Natural Science Foundation of Inner Mongolia Autonomous Region, China (Grant no. 2018MS01005).

\section{References}

[1] D. Shechtman, I. Blech, D. Gratias, and J. W. Cahn, "Metallic phase with long-range orientational order and no translational symmetry," Physical Review Letters, vol. 53, no. 20, pp. 1951-1953, 1984.

[2] Z. Zhang and K. Urban, "Transmission electron microscope observations of dislocations and stacking faults in a decagonal Al-Cu-Co alloy," Philosophical Magazine Letters, vol. 60, no. 3, pp. 97-102, 1989.

[3] L. H. Li and G. T. Liu, "A screw dislocation interacting with a wedge-shaped cracks in one-dimensional hexagonal quasicrystal," Acta Physica Sinica, vol. 61, no. 8, Article ID 086103, 2012.

[4] L. H. Li and G. T. Liu, "The interaction between the dislocation and elliptic notch in one-dimensional hexagonal quasicrystals," Modern Physics Letters B, vol. 23, no. 28, pp. 3397-3407, 2009.

[5] Y. Gao, B. X. Xu, B. S. Zhao, and T. C. Chang, "New general solutions of plane elasticity of one- dimensional quasicrystal," Physica Status Solidi (B), vol. 245, no. 1, pp. 20-27, 2008.

[6] X. F. Li and T. Y. Fan, "Elastic analysis of a mode II crack in a decagonal quasi-crystal," Chinese Physics, vol. 11, no. 3, pp. 266-271, 2002.

[7] J.-H. Guo and Z.-X. Lu, "Exact solution of four cracks originating from an elliptical hole in one-dimensional hexagonal quasicrystals," Applied Mathematics and Computation, vol. 217, no. 22, pp. 9397-9403, 2011.

[8] J. H. Guo and G. T. Liu, "Exact analytic solutions for an elliptic hole with asymmetric collinear cracks in a one-dimensional hexagonal quasi-crystal," Chinese Physics B, vol. 17, no. 7, pp. 2610-2620, 2008.

[9] G. T. Liu, R. P. Guo, and T. Y. Fan, "On the interaction between dislocations and cracks in one- dimensional hexagonal quasi-crystals," Chinese Physics, vol. 12, no. 10, pp. 1149-1155, 2003.

[10] F. Lou, T. Cao, T. Qin, and C. Xu, "Plane analysis for an inclusion in $1 \mathrm{D}$ hexagonal quasicrystal using the hypersingular integral equation method," Acta Mechanica Solida Sinica, vol. 32, no. 2, pp. 249-260, 2019.

[11] X. Wang and P. Schiavone, "Elastic field near the tip of an anticrack in a decagonal quasicrys-talline material," Applied Mathematics and Mechanics, vol. 41, no. 3, pp. 401-408, 2020.
[12] X. Zhao, X. Li, and S. Ding, "Two kinds of contact problems in three-dimensional icosahedral quasicrystals," Applied Mathematics and Mechanics, vol. 36, no. 12, pp. 1569-1580, 2015.

[13] Y. Li, M. Zhao, Q.-H. Qin, and C. Fan, "Analysis solution method for 3D planar crack problems of two-dimensional hexagonal quasicrystals with thermal effects," Applied Mathematical Modelling, vol. 69, pp. 648-664, 2019.

[14] X. Y. Li, P. D. Li, T. H. Wu, M. X. Shi, and Z. W. Zhu, “Threedimensional fundamental solutions for one-dimensional hexagonal quasicrystal with piezoelectric effect," Physics Letters A, vol. 378, no. 10, pp. 826-834, 2014.

[15] J. Yu, J. Guo, E. Pan, and Y. Xing, "General solutions of plane problem in one-dimensional quasicrystal piezoelectric materials and its application on fracture mechanics," Applied Mathematics and Mechanics, vol. 36, no. 6, pp. 793-814, 2015.

[16] J. Yu, J. Guo, and Y. Xing, "Complex variable method for an anti-plane elliptical cavity of one-dimensional hexagonal piezoelectric quasicrystals," Chinese Journal of Aeronautics, vol. 28, no. 4, pp. 1287-1295, 2015.

[17] J. Yang, Y.-T. Zhou, H.-L. Ma, S.-H. Ding, and X. Li, “The fracture behavior of two asymmetrical limited permeable cracks emanating from an elliptical hole in one-dimensional hexagonal quasicrystals with piezoelectric effect," International Journal of Solids and Structures, vol. 108, pp. 175-185, 2017.

[18] L.-Z. Yang, Y. Gao, E. Pan, and N. Waksmanski, "An exact solution for a multilayered two-dimensional decagonal quasicrystal plate," International Journal of Solids and Structures, vol. 51, no. 9, pp. 1737-1749, 2014.

[19] J. Guo, Z. Zhang, and Y. Xing, "Antiplane analysis for an elliptical inclusion in 1D hexagonal piezoelectric quasicrystal composites," Philosophical Magazine, vol. 96, no. 4, pp. 349-369, 2016.

[20] Y. Li, L. Yang, Y. Gao, and E. Pan, "Cylindrical bending analysis of a layered two-dimensional piezoelectric quasicrystal nanoplate," Journal of Intelligent Material Systems and Structures, vol. 29, no. 12, pp. 2660-2676, 2018.

[21] L. Zhang, J. Guo, and Y. Xing, "Nonlocal analytical solution of functionally graded multilayered one-dimensional hexagonal piezoelectric quasicrystal nanoplates," Acta Mechanica, vol. 230, no. 5, pp. 1781-1810, 2019.

[22] Y.-B. Zhou and X.-F. Li, "Two collinear mode-III cracks in onedimensional hexagonal piezoelectric quasicrystal strip," Engineering Fracture Mechanics, vol. 189, no. 15, pp. 133-147, 2018.

[23] Y.-B. Zhou and X.-F. Li, "Exact solution of two collinear cracks normal to the boundaries of a 1D layered hexagonal piezoelectric quasicrystal," Philosophical Magazine, vol. 98, no. 19, pp. 1780-1798, 2018.

[24] Y.-B. Zhou and X.-F. Li, "Fracture analysis of an infinite 1D hexagonal piezoelectric quasicrystal plate with a penny-shaped dielectric crack," European Journal of Mechanics-A/ Solids, vol. 76, pp. 224-234, 2019.

[25] Y.-B. Zhou and X.-F. Li, "A Yoffe-type moving crack in onedimensional hexagonal piezoelectric quasicrystals," Applied Mathematical Modelling, vol. 65, pp. 148-163, 2019.

[26] L. Li and G. Liu, "Study on a straight dislocation in an icosahedral quasicrystal with piezoelectric effects," Applied Mathematics and Mechanics, vol. 39, no. 9, pp. 1259-1266, 2018.

[27] L. H. Li, X. W. Cui, and J. H. Guo, "Interaction between a screw dislocation and an elliptical hole with two asymmetrical cracks in a one-dimensional hexagonal quasicrystal with piezoelectric effect," Applied Mathematics and Mechanics, 2020. 
[28] K. Hu, H. Jin, Z. Yang, and X. Chen, "Interface crack between dissimilar one-dimensional hexagonal quasicrystals with piezoelectric effect," Acta Mechanica, vol. 230, no. 7, pp. 2455-2474, 2019.

[29] S. M. Ohr, S. J. Chang, and R. Thomson, "Elastic interaction of a wedge crack with a screw dislocation," Journal of Applied Physics, vol. 57, no. 6, pp. 1839-1843, 1998.

[30] T. Y. Zhang, P. Tong, H. Ouyang, and S. Lee, "Interaction of an edge dislocation with a wedge crack," Journal of Applied Physics, vol. 78, no. 8, pp. 4873-4880, 1995.

[31] B. J. Chen, Z. M. Xiao, and K. M. Liew, "A screw dislocation in a piezoelectric bi-material wedge," International Journal of Engineering Science, vol. 40, no. 15, pp. 1665-1685, 2002.

[32] B. J. Chen, Z. M. Xiao, and K. M. Liew, "A piezoelectric screw dislocation near a wedge-shaped bi-material interface," International Journal of Solids and Structures, vol. 40, no. 9, pp. 2041-2056, 2003.

[33] L. H. Li and Y. Zhao, "Interaction of a screw dislocation with interface and wedge-shaped cracks in one-dimensional hexagonal piezoelectric quasicrystal biomaterial," Mathematical Problems in Engineering, vol. 2019, Article ID 1037297, 7 pages, 2019.

[34] L. J. Jiang and G. T. Liu, "The interaction between a screw dislocation and wedge-shaped crack in one-dimensional hexagonal piezoelectric quasicrystal," Chinese Physics B, vol. 26, no. 4, Article ID 044601, 2017.

[35] I.-H. Lin and R. Thomson, "Cleavage, dislocation emission, and shielding for cracks under general loading," Acta Metallurgica, vol. 34, no. 2, pp. 187-206, 1986.

[36] R. R. Juang and S. Lee, "Elastic interaction between general parallel screw dislocations and a surface crack," Journal of Applied Physics, vol. 59, no. 10, pp. 3421-3429, 1986.

[37] G. T. Liu and L. Y. Yang, "Interaction between infinitely many dislocations and a semi-infinite crack in one-dimensional hexagonal quasicrystal," Chinese Physics B, vol. 26, no. 9, Article ID 094601, 2017.

[38] X. Lv and G. T. Liu, "Interaction between many parallel screw dislocations and a semi-infinite crack in a magnetoelectroelastic solid," Chinese Physics B, vol. 27, no. 7, Article ID 074601, 2018.

[39] G. Altay and M. C. Dökmeci, "On the fundamental equations of piezoelasticity of quasicrystal media," International Journal of Solids and Structures, vol. 49, no. 23-24, pp. 3255-3262, 2012.

[40] N. I. Muskhelishvili, Singular Integral Equations, Noordhoff, Groningen, Holland, 1953.

[41] R. J. Hao and J. X. Liu, "Interaction of a screw dislocation with a semi-infinite interfacial crack in a magneto-electro-elastic bi-material," Mechanics Research Communications, vol. 33, no. 3, pp. 415-424, 2006.

[42] K. Y. Lee, W. G. Lee, and Y. E. Pak, "Interaction between a semi-infinite crack and a screw dislocation in a piezoelectric material," Journal of Applied Mechanics, vol. 67, no. 1, pp. 165-170, 2000.

[43] Y. E. Pak, "Crack extension force in a piezoelectric material," Journal of Applied Mechanics, vol. 57, no. 3, pp. 647-653, 1990. 